\title{
Meteorización y marco tectónico de rocas siliciclásticas de la Formación Morita, noreste de Sonora, México
}

\author{
Erik Ramírez-Montoya ${ }^{1}$, Jayagopal Madhavaraju ${ }^{2, *}$, Rogelio Monreal $^{1}$, \\ Carlos M. González-León ${ }^{2}$, Francisco J. Grijalva-Noriega ${ }^{1}$, \\ Julio Cesar Saucedo-Samaniego ${ }^{3}$, Inocente G. Espinoza-Maldonado ${ }^{1}$ \\ ${ }^{1}$ Departamento de Geología, Universidad de Sonora, Hermosillo, Sonora 83000, México. \\ ${ }^{2}$ Estación Regional del Noroeste, Instituto de Geología, Universidad Nacional Autónoma de México, \\ Hermosillo, Sonora 83000, México. \\ ${ }^{3}$ Posgrado en Ciencias de la Tierra, Estación Regional del Noroeste, Instituto de Geología, \\ Universidad Nacional Autónoma de México, Hermosillo, Sonora 83000, México. \\ *mj@geologia.unam.mx
}

\section{RESUMEN}

En el presente trabajo se hace una interpretación de las condiciones de meteorización y del marco tectónico del área fuente de las rocas siliciclásticas de la Formación Morita (Cretácico Inferior) a partir de datos de geoquímica de óxidos mayores obtenidos en muestras colectadas de sus columnas de la Sierra Anibacachi y el área de Rancho Búfalo, en noreste de Sonora. La Formación Morita es una sucesión siliciclástica de origen fluvial que alcanza un espesor de $560 \mathrm{~m}$ en la Sierra Anibacachi y $855 \mathrm{~m}$ en el área de Rancho Búfalo. Geoquímicamente estas rocas se clasifican como sub-litarenita, litarenita, subarcosa y arenas-Fe. Los valores de CIA y PIA junto con el diagrama A-CN-K de las areniscas de la sección Sierra Anibacachi indican una meteorización química de baja a intensa del área de origen, mientras que los valores de CIA para limolitas y lutitas muestran una intensidad moderada en el área de origen. De igual manera, los valores de CIA, PIA y el diagrama A-CN-K sugieren que las areniscas de la sección Rancho Búfalo derivaron de un área de origen con meteorización baja a intensa, mientras que las limolitas provienen de una región con meteorización baja a moderada. Las rocas siliciclásticas de las secciones Sierra Anibacachi y Rancho Búfalo muestran grandes variaciones en valores de ICV, indicando que composicionalmente varían de inmaduras a maduras. El diagrama discriminatorio tectónico basado en función multidimensional muestra campos de colisión, arco y rift para las areniscas de la sección Sierra Anibacachi, y colisión y rift para las limolitas, mientras que para las lutitas indicó colisión. Sin embargo, la mayoría de las areniscas y limolitas de la sección Rancho Búfalo se representan en los gráficos en el campo de arco, pero algunas areniscas se ubican en el campo de colisión. Las rocas silicilásticas de las secciones Sierra Anibacachi y Rancho Búfalo muestran gran variación en los ambientes tectónicos de las rocas de origen, sin embargo, los resultados obtenidos de los diagramas de función discriminante son consistentes con la evolución tectónica regional del área de estudio.

Palabras clave: geoquímica; paleointemperismo; ambiente tectónico; Formación Morita; Sonora, México.

\begin{abstract}
Geochemistry of major oxides was carried out on the siliciclastic rocks collected from the Sierra Anibacachi and Rancho Búfalo sections of the Morita Formation (Early Cretaceous) exposed in the northeast of Sonora, to interpret the paleoweathering conditions and tectonic settings of the source rocks. Geochemically, the siliciclastic rocks were classified as sublitharenite, litharenite, subarkose and Fe-sand. The Morita Formation consists mainly of siliciclastic rocks that were deposited under fluvial conditions and the total thickness vary between 560 and $855 \mathrm{~m}$ in Sierra Anibacahi and Rancho Búfalo, respectively. The CIA and PIA values and A-CN-K diagram of sandstones from Sierra Anibacachi section indicate a low to intense chemical weathering of the source area, whereas CIA values for siltstone and shale show moderate intensity of chemical weathering in the source region. Similarly, the CIA and PIA values and the A-CN-K plot suggest that the sandstones from Rancho Búfalo section were derived from low to intensely weathered source region, whereas siltstones were received sediments from source rocks affected by low to moderate degree of chemical weathering. The siliciclastic rocks from Sierra Anibacachi and Rancho Búfalo sections show large variations in ICV values, indicating that these samples are compositionally immature to mature. The tectonic discriminant-function-based multidimensional diagram shows arc, rift
\end{abstract}

Ramírez-Montoya, E., Madhavaraju, J., Monreal, R., González-León, C.M., Grijalva-Noriega, F.J., Saucedo-Samaniego, J.C., Espinoza-Maldonado, I.G., 2018, Meteorización y marco tectónico de rocas siliciclásticas de la Formación Morita, noreste de Sonora, México: Revista Mexicana de Ciencias Geológicas, v. 35, núm. 2, p. 103-115. 
and collision fields for the sandstones from Sierra Anibacachi section, rift and collision for siltstone, while collision setting for shales. However, most of the sandstones and siltstones from Rancho Búfalo section fall in the arc field, whereas some sandstones fall in the collision field. The siliciclastic rocks from Sierra Anibacachi and Rancho Búfalo section show large variations in the tectonic settings of the source rocks. However, the results obtained from the discriminant function diagrams are consistent with a regional tectonic evolution of the study area.

Key words: geochemistry; paleoweathering; tectonic settings; Morita Formation; Sonora; Mexico.

\section{INTRODUCCIÓN}

Las rocas sedimentarias siliciclásticas proporcionan abundante información sobre la composición, marco tectónico y el desarrollo evolutivo de la corteza continental (Taylor y McLennan, 1985; McLennan et al., 1993). Las unidades sedimentarias arenosas son parte importante de las cuencas sedimentarias en todo el mundo y constituyen reservorios potenciales en la mayoría de las provincias petroleras. Las características texturales de tales unidades son principalmente producto de la meteorización del área fuente y del transporte y procesos sedimentarios asociados, mientras que su composición depende de la mineralogía primaria de las rocas del área fuente y del marco tectónico de la cuenca de depósito (Bhatia y Crook, 1986; Das y Haake, 2003; Jin et al., 2006). Por lo tanto, la geoquímica de las rocas sedimentarias siliciclásticas ha sido empleada como un indicador sensible para determinar las condiciones de meteorización del área fuente y procedencia de los sedimentos (Roser y Korsch, 1986, 1988; Goetze, 1998; Cullers, 2000; Getaneh, 2002; Ohta, 2004; Huntsman-Mapilaa et al., 2005; Madhavaraju y Lee, 2010; Madhavaraju, 2015; Armstrong-Altrin et al., 2014, 2016; Tapia-Fernandez, et al., 2017).

La composición geoquímica de las rocas clásticas también se ha usado ampliamente para descifrar la composición del área fuente (Wronkiewicz y Condie, 1987, 1989, 1990; McLennan et al., 1995; Cullers, 2000; Cullers y Podkovyrov, 2000; Bhat y Ghosh, 2001; Condie et al., 2001; Chakrabarti et al., 2007a, 2017b; Armstrong-Altrin et al., 2013; Madhavaraju, 2015; Madhavaraju et al., 2016a; Ramachandran et al., 2016), para evaluar procesos de meteorización y paleoclimas (e.g. Nesbitt y Young, 1982; Sreenivas y Srinivasan, 1994; Fedo et al., 1995, 1996; Madhavaraju et al., 2016b) , para reconstruir los marcos tectónicos de las cuencas (e.g. Bhatia, 1983; Bhatia y Crook, 1986; Roser y Korsch, 1986, 1988; McLennan et al., 1990; Verma y Armstrong-Altrin, 2013, 2016), para cuantificar los procesos secundarios como metasomatismo postdepositacional (e.g. Fedo et al., 1995, 1997) y también para evaluar la composición y evolución de la corteza continental (e.g. Taylor y McLennan, 1985, 1995; Gibbs et al., 1986; McLennan y Taylor, 1991; Condie, 1993; Lahtinen, 2000; Rudnick y Gao, 2004).

La geoquímica de las lutitas ofrece mayor información con relación a la composición promedio de la corteza continental superior, ya que conserva la firma original de su procedencia e historia diagenética (Baioumy e Ismael, 2010; DaPeng et al., 2012; Mondal et al., 2012; Spalletti et al., 2012). La composición geoquímica de las rocas clásticas de grano fino ha sido usada para interpretar las características de la roca fuente, la intensidad de la meteorización y el marco tectónico (Bhatia, 1983; Taylor y McLennan, 1985; Bhatia y Crook, 1986; Feng y Kerrich, 1990; Cullers, 1994; Madhavaraju y Lee, 2010; Armstrong-Altrin et al., 2013; Madhavaraju, 2015; Tobia y Shangola, 2016).

El presente artículo presenta el estudio geoquímico de óxidos mayores llevado a cabo en rocas siliciclásticas de las columnas de la Formación Morita en las áreas de Sierra Anibacachi y Rancho Búfalo en el noreste de Sonora, México (Figura 1). La Formación Morita es parte del Grupo Bisbee (Ransome, 1904) y los objetivos principales de este trabajo son interpretar las variaciones en las condiciones de meteorización del área fuente que dieron origen a los sedimentos que componen a la Formación Morita, así mismo, inferir el marco tectónico de las rocas de las regiones de procedencia.

\section{ESTRATIGRAFÍA}

Las formaciones sedimentarias que componen al Grupo Bisbee están bien expuestas en el noreste de Sonora y el sureste de Arizona. Desde la base a la cima, las rocas del Grupo Bisbee se conforman por el Conglomerado Glance, Formación Morita, Caliza Mural y Formación Cintura. Estas unidades fueron depositadas en la Cuenca Bisbee (Dickinson et al., 1986) durante el Jurásico Superior y el Cretácico Inferior y representan ambientes aluviales, fluviales y marinos someros. En el noreste de Sonora, el Grupo Bisbee sobreyace discordantemente a rocas volcánicas y sedimentarias del arco continental Jurásico Nazas (Busby-Spera, 1988; Busby-Spera et al., 1990; Lawton y Molina-Garza, 2014) y a una secuencia Paleozoica que a su vez descansa sobre un basamento paleoproterozoico formado por el Esquisto Pinal y por granitos mesoproterozoicos que lo cortan (Anderson y Silver, 2005; Page et al., 2010; Solari et al., 2017).

La Formación Morita, objeto de este estudio, consiste en una secuencia clástica con intercalaciones de tobas volcánicas que fue depositada durante el Cretácico Inferior y la forman principalmente secuencias grano-decreciente, superpuestas, de origen fluvial compuestas por areniscas, limolitas, lutitas y escasos conglomerados. El espesor total de esta formación varía de 280 a 1200 m en Sonora y su estratigrafía ha sido estudiada por varios autores en diversas areas (Taliaferro, 1933; Jaques-Ayala, 1992, 1995; González-León, 1994; Monreal et al., 1994; Jacques-Ayala, 1995; Peryam et al., 2012). Escasos

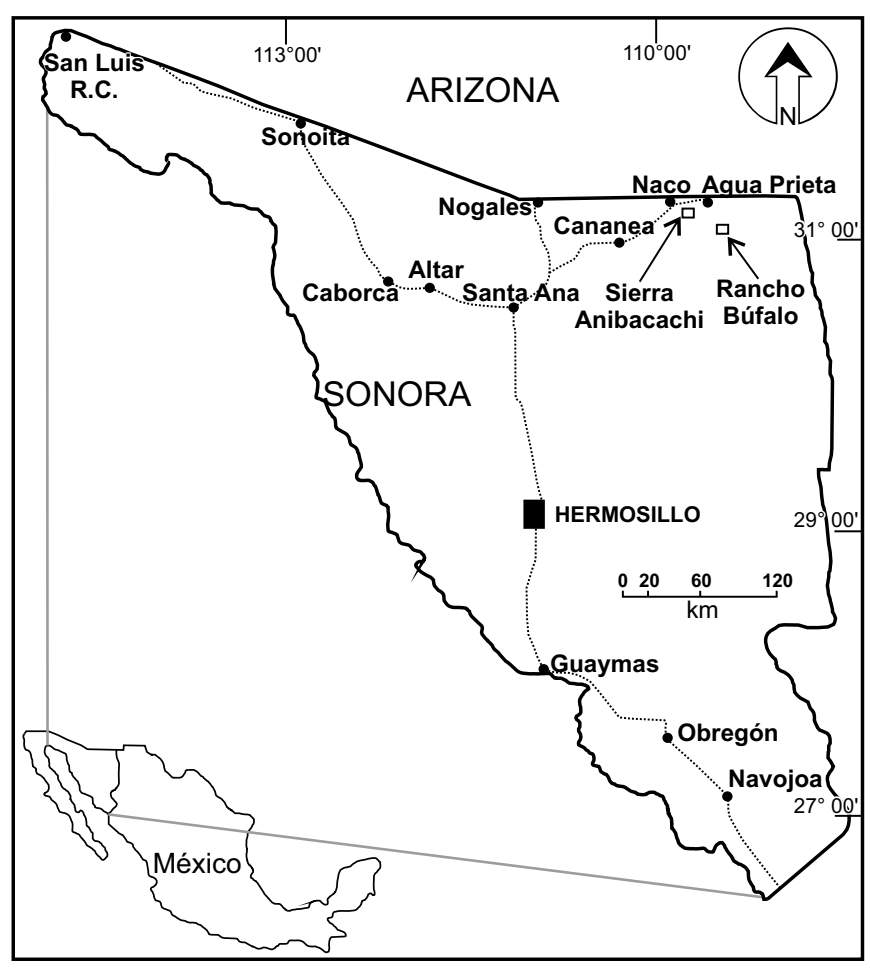

Figura 1. Mapa de localización del área de estudio. 
estudios locales de direcciones de paleocorrientes y procedencia en las áreas de Sierra El Chanate, Cerro de Oro y Tuape del norponiente y centro de Sonora indican que la Formacion Morita fue depositada por sistemas de ríos meándriformes con dirección de transporte hacia el este desde fuentes volcánicas localizadas al oeste de la cuenca Bisbee (González-León, 1994; Peryam et al., 2012).

\section{Estratigrafía de la Formación Morita en la Sierra Anibacachi}

La Formación Morita, que aflora en la parte norte de la Sierra Anibacachi, tiene un espesor de $560 \mathrm{~m}$ y sobreyace abruptamente un paquete de lodolitas-limolitas moradas con abundantes capas y nódulos calcáreos que representan paleosuelos de la parte superior del Conglomerado Glance (Figura 2). En su parte superior la Formación
Morita pasa gradualmente a lutitas calcáreas y calizas con bivalvos marinos de la Caliza Mural. La Formación Morita en esta localidad está formada por ciclos superpuestos de origen fluvial que tienen espesores de 15 a $60 \mathrm{~m}$ (Figura 3). La parte inferior de cada ciclo se compone de un paquete de arenisca de hasta $10 \mathrm{~m}$ de espesor, con base erosiva, donde las capas mayores a $1 \mathrm{~m}$ de espesor tienen comúnmente estratificación cruzada y planar. Una capa lenticular de conglomerado, de menos de $80 \mathrm{~cm}$ de espesor, puede a veces estar presente en la parte basal del ciclo. Encima de las areniscas aparecen paquetes de lutitas y limolitas de hasta $50 \mathrm{~m}$ de espesor, comúnmente de colores rojizos a púrpuras y con perforaciones y rastros de organismos que producen bioturbación local. Dentro de las lutitas y limolitas se intercalan arenisca de grano fino a medio, con laminación paralela, a veces biotur-
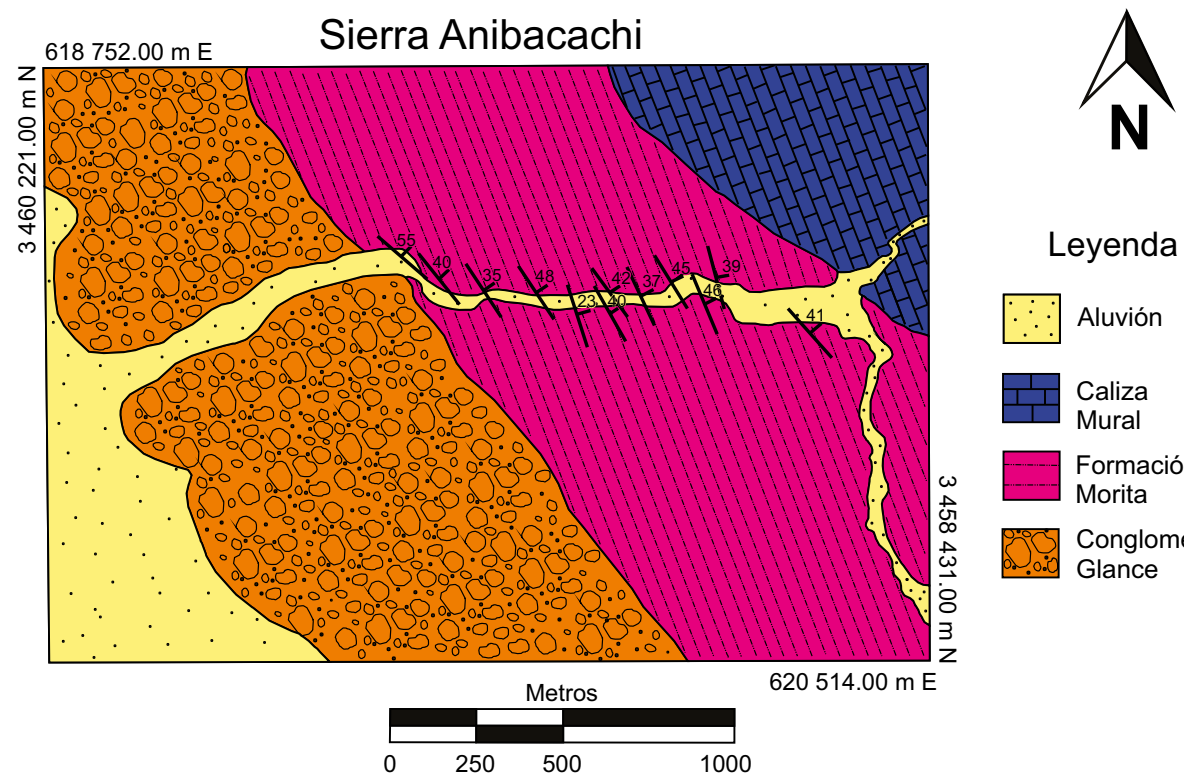

\section{Leyenda}

Aluvión

I Caliza

Mural

Formación

Morita

एọ Conglomerado

Glance
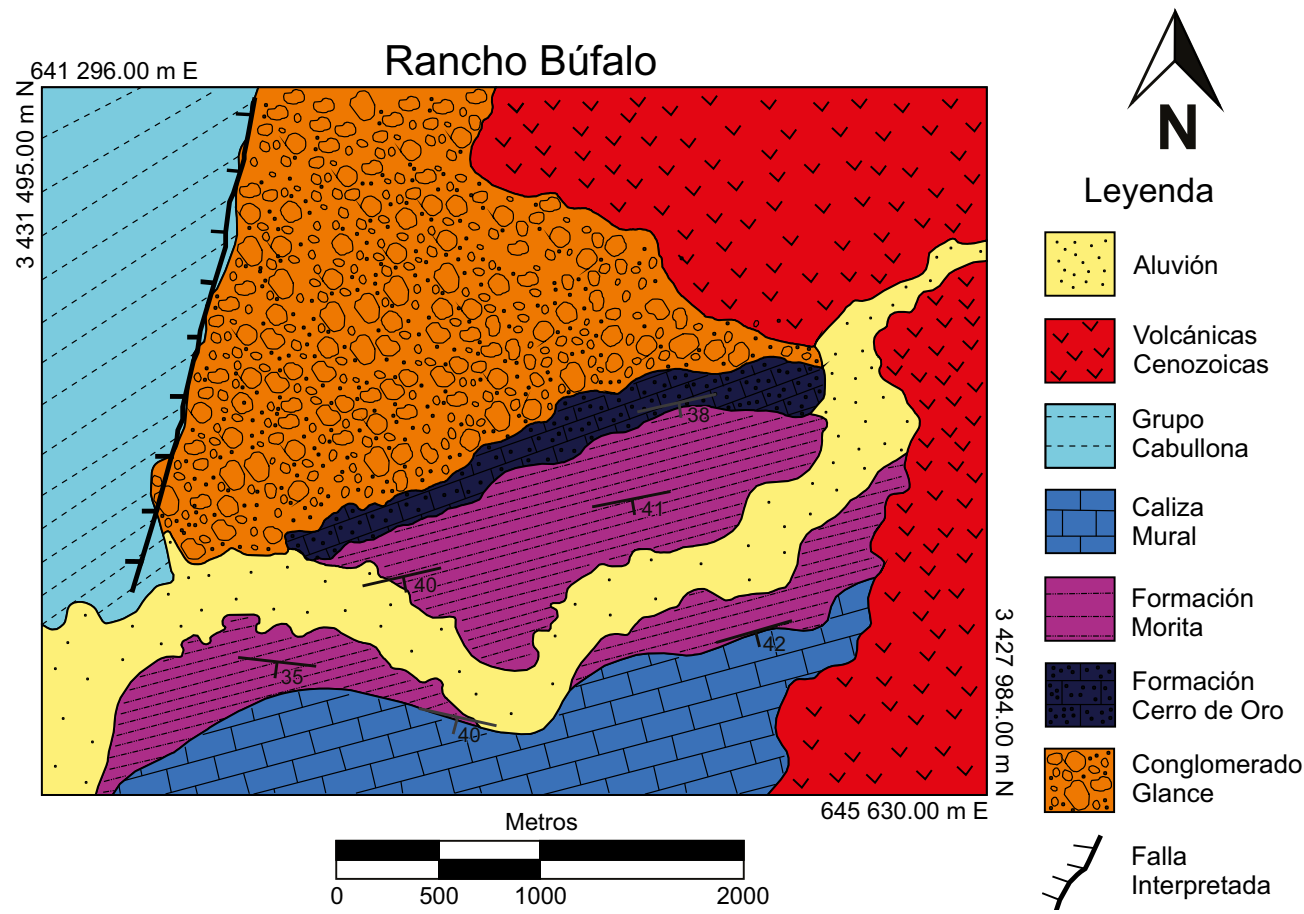

Figura 2. Mapas geológicos de las áreas de Sierra Anibacachi (arriba) y Rancho Búfalo (abajo). 

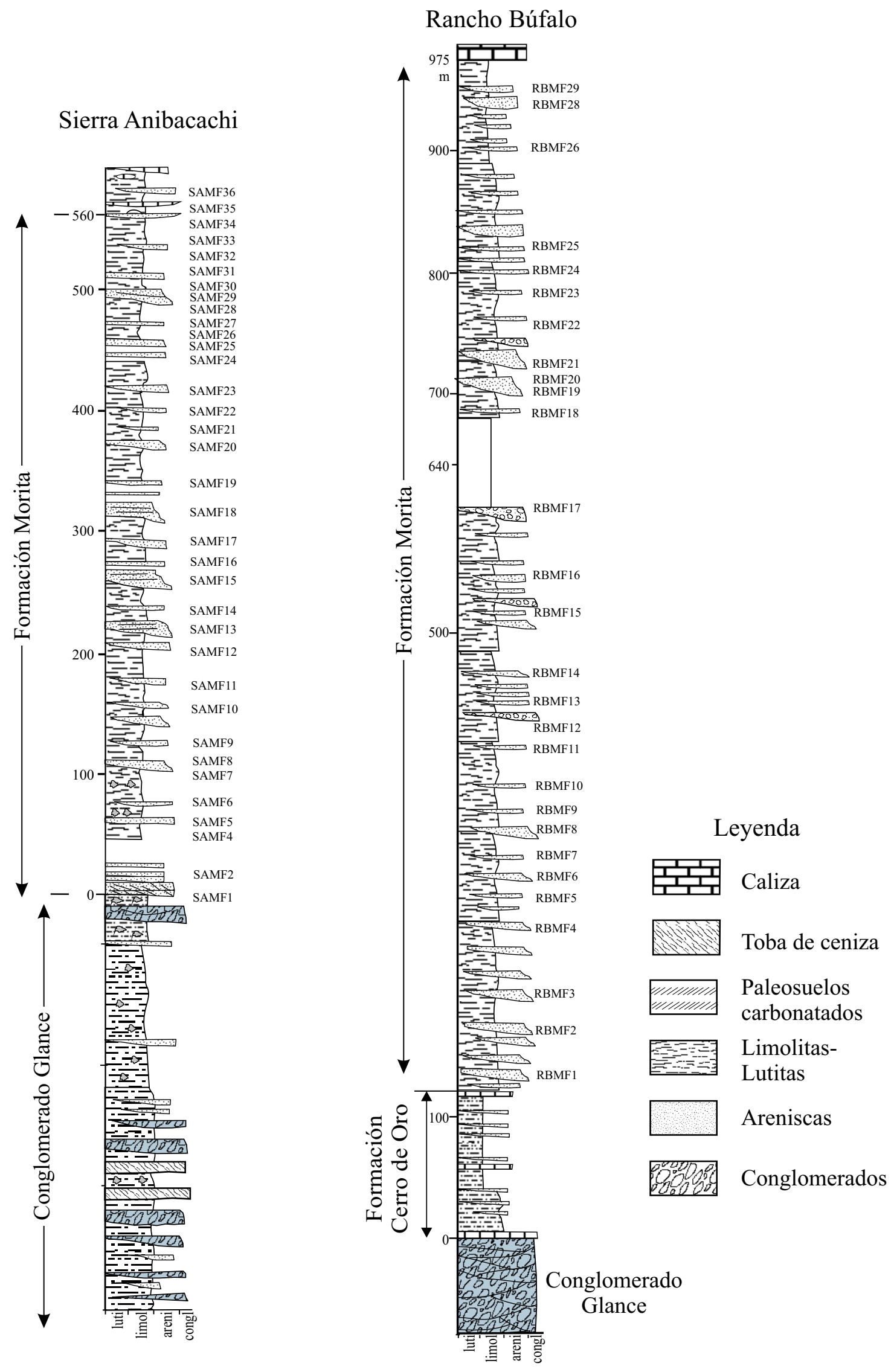

Figura 3. Columna litoestratigráfica de la Formación Morita en las secciones Sierra Anibacachi y Rancho Búfalo. 
badas que ocurren en capas de hasta $50 \mathrm{~cm}$ de espesor. Intercalados dentro de las limolitas-lutitas también se tienen abundantes nódulos calcáreos de origen pedogénico en niveles de hasta $50 \mathrm{~cm}$ de espesor. Los 145 m más superiores de esta formación en la Sierra Anibacachi, están dominados por lutitas y limolitas púrpuras, rojizas y verdosas, masivas a localmente laminadas, que tienen intercalaciones de capas lenticulares de areniscas de granos finos menores a $40 \mathrm{~cm}$ de espesor, ocasionales nódulos calcáreos pedogénicos y restos de madera fósil.

\section{Estratigrafía de la Formación Morita en Rancho Búfalo}

La Formación Morita en la sección Rancho Búfalo tiene $855 \mathrm{~m}$ de espesor y se superpone a un paquete de $120 \mathrm{~m}$ de espesor de lutitas, limolitas y calizas bioclásticas que a su vez sobreyace discordantemente al Conglomerado Glance. Este paquete intermedio puede ser correlacionable con las formaciones equivalentes, Cerro de Oro y La Colgada (Figura 2) que afloran en la parte suroccidental de la cuenca Bisbee en Sonora (Peryam et al., 2012). La parte superior de la Formación Morita se encuentra en contacto gradacional con calizas bioclásticas del Miembro Rancho Búfalo de la Caliza Mural.

En su parte basal la Formación Morita consiste en un intervalo de $5 \mathrm{~m}$ de espesor de areniscas de grano grueso a conglomeráticas que gradúan hacia arriba a paquetes de $10 \mathrm{~m}$ de espesor de lutitaslimolitas masivas púrpuras con intercalaciones menores de capas de areniscas de grano medio a grueso (Figura 3). El resto de la formación lo forman ciclos fluviales superpuestos de 5 a $130 \mathrm{~m}$ de espesor que consisten de capas lenticulares de conglomerados con base erosiva que están sobreyacidas por niveles de hasta $10 \mathrm{~m}$ de arenisca con estratificación cruzada. Estas últimas gradúan hacia arriba a paquetes de lutitas-limolitas masivas a localmente laminadas, rojizas a púrpura y que se vuelven de colores gris a verdosos hacia la parte superior de la formación. Intercaladas con las lutitas-limolitas se tienen capas lenticulares, delgadas a medianas de areniscas rojizas de grano medio a fino que presentan estratificación cruzada planar, laminación paralela y escasa bioturbación. En la parte inferior de la formación se tienen varias capas de tobas de ceniza que tienen espesores de hasta $20 \mathrm{~cm}$.

\section{MATERIALES Y MÉTODOS}

Durante el presente estudio se analizaron 62 muestras de areniscas, limolitas y lutitas para determinar su composición química en óxidos mayores. De éstas, 35 muestras corresponden a la columna de la Sierra Anibacachi y 27 fueron colectadas de la columna de Rancho Búfalo. Además, también fueron analizadas dos muestras del Esquisto Pinal y cuatro del granito Mesteñas con el fin de caracterizar geoquímicamente las rocas que en la región noroeste de Sonora constituyen el basamento Proterozoico. Las muestras fueron pulverizadas en un mortero de ágata y fundidas en perlas de vidrio para el análisis de elementos mayores. Esta medición se realizó por medio de la técnica de fluorescencia de rayos-X en discos fusionados de $\mathrm{LiBO}_{2} / \mathrm{Li}_{2} \mathrm{~B}_{4} \mathrm{O}_{7}$, utilizando un espectrómetro de fluorescencia de rayos-X Siemens SRS-3000 con un tubo con ánodo de Rh como fuente de radiación. Los efectos de absorción/ realce fueron corregidos usando el método de Lachance y Traill (1966), incluido en el software SRS-3000. Se utilizó el estándar geoquímico JGB1 (GSJ) para determinar la calidad de los análisis químicos. La precisión de los análisis presenta errores inferiores al $\pm 2 \%$ para $\mathrm{SiO}_{2}$, $\mathrm{Fe}_{2} \mathrm{O}_{3}$ y CaO (1.24\%, $0.73 \%, 1.52 \%$, respectivamente) y menores al $\pm 5 \%$ para $\mathrm{Al}_{2} \mathrm{O}_{3}, \mathrm{MgO}$ y $\mathrm{Na}_{2} \mathrm{O}$ (3.61\%, 3.44\%, 3.33\%, respectivamente). Las precisiones para $\mathrm{MnO}$ y $\mathrm{P}_{2} \mathrm{O}_{5}$ presenta errores inferiores al $\pm 5 \%(5.27 \%$, $7.14 \%$, respectivamente). Un gramo de muestra fue calentada a $1000^{\circ} \mathrm{C}$ en crisol de porcelana durante 1 hora para medir la pérdida por ignición (LOI: Loss on ignition, por sus siglas en inglés).

\section{RESULTADOS}

\section{Sección Sierra Anibacachi}

Las areniscas de esta sección tienen una mayor concentración de $\mathrm{SiO}_{2}(62.5$ a $94.6 \%)$ que las limolitas y lutitas (56.1 a 67.7\%, 49.9 a $65.8 \%$, respectivamente). En cambio, las limolitas y lutitas tienen mayor contenido en $\mathrm{Al}_{2} \mathrm{O}_{3}$ (6.41 a 8.86\%, 9.88 a $17.05 \%$, respectivamente) que los valores obtenidos en las areniscas (1.81 a 7.03\%). Las areniscas y lutitas muestran variaciones más grandes en los contenidos en $\mathrm{CaO}$ (0.05-15.50\%; 0.63-15.35\%; respectivamente) que las limolitas (9.09-15.27\%). Las areniscas y limolitas tienen contenidos más bajos de $\mathrm{TiO}_{2}, \mathrm{MgO}, \mathrm{Na}_{2} \mathrm{O}$ y $\mathrm{K}_{2} \mathrm{O}$ que las lutitas. Por último, las areniscas, limolitas y lutitas tienen bajo contenido de $\mathrm{MnO}$ y $\mathrm{P}_{2} \mathrm{O}_{5}$ (Tabla 1). En el diagrama de Herron (1988) $\log \left(\mathrm{Fe}_{2} \mathrm{O}_{3} / \mathrm{K}_{2} \mathrm{O}\right)$ vs $\mathrm{Log}\left(\mathrm{SiO}_{2} / \mathrm{Al}_{2} \mathrm{O}_{3}\right)$, Figura 4a, la mayoría de las muestras de areniscas caen dentro de los campos sub-litarenita, litarenita y sub-arcosa, mientras que las muestras de lutita se encuentran en los campos de wacka y lutita. La relación $\mathrm{K}_{2} \mathrm{O} / \mathrm{Al}_{2} \mathrm{O}_{3}$ de las rocas sedimentarias terrígenas puede ser usada como un indicador de la composición original de sedimentos antiguos, porque dicha relación es diferente para minerales de arcilla y feldespatos. Continuando con la relación anterior, el rango de valores para minerales de arcilla es de 0.0 a 0.03 y para feldespatos el rango se encuentra entre
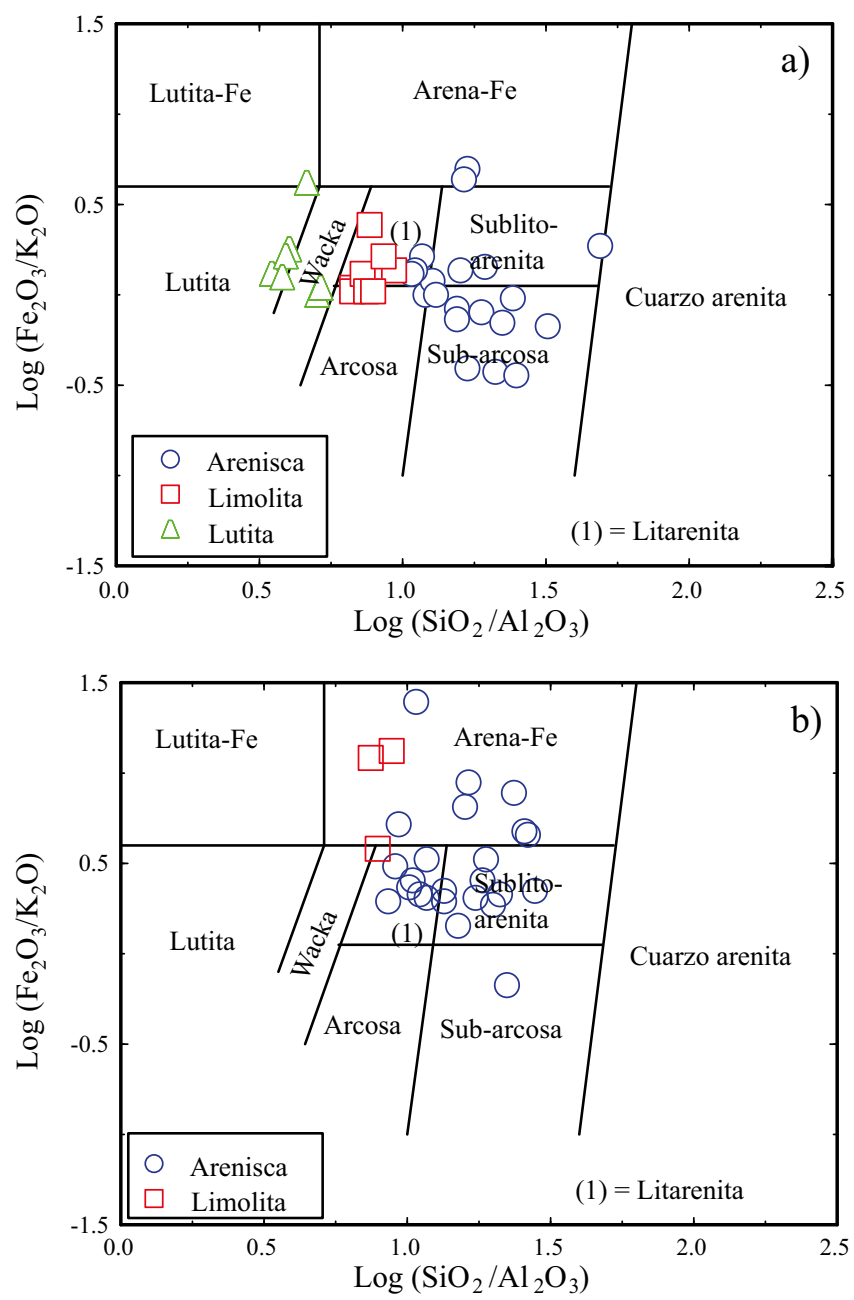

Figura 4. a) Clasificación geoquímica de areniscas, limolitas y lutitas de la sección Sierra Anibacachi y b) Clasificación geoquímica de areniscas, limolitas y lutitas de la sección Rancho Búfalo utilizando diagramas $\log \left(\mathrm{SiO}_{2} / \mathrm{Al}_{2} \mathrm{O}_{3}\right)$ $\mathrm{Log}\left(\mathrm{Fe}_{2} \mathrm{O}_{3} / \mathrm{K}_{2} \mathrm{O}\right.$ ) (Herron, 1988). 
Tabla 1. Datos de óxidos mayores (wt\%) para areniscas, limolitas y lutitas de la Formación Morita en la sección Sierra Anibacachi.

\begin{tabular}{|c|c|c|c|c|c|c|c|c|c|c|c|c|c|c|c|c|c|c|c|}
\hline $\begin{array}{l}\text { Muestra } \\
\text { Núm. }\end{array}$ & $\mathrm{SiO}_{2}$ & $\mathrm{TiO}_{2}$ & $\mathrm{Al}_{2} \mathrm{O}_{3}$ & $\mathrm{Fe}_{2} \mathrm{O}_{3}$ & $\mathrm{MnO}$ & $\mathrm{MgO}$ & $\mathrm{CaO}$ & $\mathrm{Na}_{2} \mathrm{O}$ & $\mathrm{K}_{2} \mathrm{O}$ & $\mathbf{P}_{2} \mathbf{O}_{5}$ & PPC & Suma & CIA & PIA & ICV & $\begin{array}{l}\mathrm{SiO}_{2} / \\
\mathrm{Al}_{2} \mathrm{O}_{3}\end{array}$ & $\begin{array}{r}\mathrm{K}_{2} \mathrm{O} / \\
\mathrm{Al}_{2} \mathrm{O}_{3} \\
\end{array}$ & $\begin{array}{l}\mathrm{K}_{2} \mathrm{O} / \\
\mathrm{Na}_{2} \mathrm{O} \\
\end{array}$ & $\begin{array}{r}\mathrm{Al}_{2} \mathrm{O}_{3} / \\
\mathrm{TiO}_{2}\end{array}$ \\
\hline \multicolumn{20}{|l|}{ Arenisca } \\
\hline SAMF2 & 90.07 & 0.17 & 5.66 & 0.41 & 0.03 & 0.39 & 0.11 & 1.57 & 0.31 & 0.06 & 1.2 & 99.99 & 64 & 66 & 0.53 & 15.9 & 0.06 & 0.2 & 33 \\
\hline SAMF5 & 81.68 & 0.21 & 5.14 & 0.8 & 0.03 & 0.24 & 5.16 & 0.8 & 0.98 & 0.03 & 4.88 & 99.95 & 58 & 61 & 1.6 & 15.9 & 0.19 & 1.22 & 24 \\
\hline SAMF6 & 69.63 & 0.35 & 5.72 & 1.29 & 0.07 & 0.33 & 11 & 0.48 & 1.31 & 0.06 & 9.62 & 99.88 & 66 & 73 & 2.6 & 12.2 & 0.23 & 2.72 & 16 \\
\hline SAMF8 & 74.02 & 0.15 & 4.75 & 0.54 & 0.08 & 0.22 & 9.85 & 1.01 & 0.75 & 0.03 & 8.38 & 99.78 & 54 & 54 & 2.65 & 15.6 & 0.16 & 0.74 & 33 \\
\hline SAMF10 & 81.84 & 0.14 & 4.82 & 0.62 & 0.05 & 0.21 & 5.29 & 2.17 & 0.13 & 0.03 & 4.69 & 99.98 & 40 & 40 & 1.78 & 17 & 0.03 & 0.06 & 34 \\
\hline SAMF11 & 62.49 & 0.2 & 5.26 & 0.72 & 0.18 & 0.33 & 15.5 & 2.23 & 0.45 & 0.04 & 12.58 & 99.98 & 40 & 39 & 3.73 & 11.9 & 0.08 & 0.2 & 27 \\
\hline SAMF13 & 68.53 & 0.29 & 5.35 & 1.24 & 0.09 & 0.39 & 11.82 & 0.91 & 1.06 & 0.04 & 10.25 & 99.95 & 56 & 58 & 2.95 & 12.8 & 0.2 & 1.16 & 19 \\
\hline SAMF14 & 83.13 & 0.16 & 4.3 & 0.33 & 0.03 & 0.12 & 4.95 & 1.54 & 0.43 & 0.04 & 4.29 & 99.31 & 44 & 43 & 1.76 & 19.3 & 0.1 & 0.28 & 27 \\
\hline SAMF17 & 77.63 & 0.35 & 7.03 & 2.05 & 0.07 & 0.31 & 4.8 & 1.04 & 1.54 & 0.09 & 5.03 & 99.95 & 58 & 61 & 1.44 & 11 & 0.22 & 1.48 & 20 \\
\hline SAMF18 & 88.08 & 0.29 & 5.21 & 0.32 & 0.02 & 0.17 & 1.65 & 1.18 & 0.83 & 0.04 & 2.13 & 99.92 & 5 & 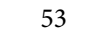 & 0.86 & 16.9 & 0.16 & 0.7 & 18 \\
\hline SAMF20 & 86.14 & 0.23 & 4.4 & 0.8 & 0.04 & 0.19 & 3.21 & 1.24 & 0.58 & 0.03 & 3.11 & 99.97 & 48 & 48 & 1.43 & 19.6 & 0.13 & 0.47 & 19 \\
\hline SAMF22 & 74.65 & 0.42 & 6.89 & 2.07 & 0.06 & 0.95 & 5.85 & 0.87 & 1.63 & 0.05 & 6.5 & 99.95 & 60 & 6 & 1.72 & 10.8 & 0.24 & 1.88 & 16 \\
\hline SAMF-23 & 91.95 & 0.41 & 4.05 & 0.56 & 0.01 & 0.27 & 0.32 & 0.02 & 0.84 & 0.06 & 1.32 & 99.81 & 80 & 9 & 0.6 & 22.7 & 0.21 & 34.79 & 10 \\
\hline SAMF24 & 68.76 & 0.34 & 5.13 & 1.14 & 0.09 & 0.39 & 11.99 & 0.51 & 1.14 & 0.04 & 10.44 & 99.95 & 64 & 70 & 3.04 & 13.4 & 0.22 & 2.22 & 15 \\
\hline SAMF25 & 91.44 & 0.42 & 4.33 & 0.32 & 0.03 & 0.17 & 0.63 & 0.1 & 0.87 & 0.04 & 1.47 & 99.83 & 77 & 91 & 0.59 & 21.1 & 0.2 & 8.56 & 10 \\
\hline SAMF27 & 94.36 & 0.13 & 3.69 & 0.15 & 0.01 & 0.13 & 0.05 & 0.01 & 0.42 & 0.02 & 1.04 & 99.99 & 88 & 99 & 0.24 & 25.6 & 0.11 & 60.29 & 28 \\
\hline SAMF28 & 90.3 & 0.31 & 3.69 & 0.47 & 0.05 & 0.28 & 1.49 & 0.59 & 0.5 & 0.04 & 2.22 & 99.93 & 60 & 6 & 1 & 24.5 & 0.14 & 0.85 & 12 \\
\hline SAMF29 & 94.6 & 0.24 & 2.94 & 0.24 & 0.01 & 0.18 & 0.08 & 0.02 & 0.37 & 0.03 & 1.26 & 99.96 & 86 & 98 & 0.39 & 32.2 & 0.13 & 21.88 & 12 \\
\hline SAMF30 & 88.76 & 0.06 & 1.81 & 0.3 & 0.06 & 0.15 & 4.35 & 0.51 & 0.17 & 0.03 & 3.78 & 99.98 & 50 & 49 & 3.09 & 49 & 0.09 & 0.33 & 31 \\
\hline SAMF35 & 88.19 & 0.37 & 5.38 & 1.23 & 0.01 & 0.87 & 0.4 & 1.72 & 0.28 & 0.04 & 1.23 & 99.73 & 58 & 59 & 0.91 & 16.4 & 0.05 & 0.17 & 14 \\
\hline Promedio & $\begin{array}{r}82.3 \\
\pm 9.7\end{array}$ & $\begin{array}{r}0.3 \\
\pm 0.1\end{array}$ & $\begin{array}{r}4.7 \\
\pm 1.2\end{array}$ & $\begin{array}{r}0.7 \\
\pm 0.5\end{array}$ & $\begin{array}{r}0.05 \\
\pm 0.04\end{array}$ & $\begin{array}{r}0.3 \\
\pm 0.2\end{array}$ & $\begin{array}{l}4.9 \\
\pm \\
\pm .75\end{array}$ & 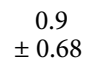 & $\begin{array}{r}0.7 \\
\pm 0.4\end{array}$ & $\begin{array}{r}0.04 \\
\pm 0.02\end{array}$ & $\begin{array}{r}4.7 \\
\pm 3.6\end{array}$ & $\begin{array}{r}99.9 \\
\pm 0.2\end{array}$ & $\begin{array}{r}60 \\
\pm 14\end{array}$ & $\begin{array}{r}64 \\
+19\end{array}$ & $\begin{array}{r}1.6 \\
\pm 1.0\end{array}$ & $\begin{array}{r}19.2 \\
\pm 8.9\end{array}$ & $\begin{array}{r}0.15 \\
+0.06\end{array}$ & $\begin{array}{r}1.37 \\
\pm 2.01\end{array}$ & $\begin{array}{r}21 \\
\pm 8\end{array}$ \\
\hline \multicolumn{20}{|l|}{ Limolita } \\
\hline SAMF1 & 56.13 & 0.31 & 8.42 & 1.84 & 0.14 & 1.14 & 15.27 & 1.14 & 1.7 & 0.03 & 13.83 & 99.93 & 60 & 64 & 2.56 & 6.7 & 0.2 & 1.5 & 28 \\
\hline SAMF4 & 60.28 & 0.42 & 8.86 & 2.68 & 0.07 & 0.39 & 12.97 & 0.06 & 2.58 & 30.06 & 11.61 & 99.98 & 75 & 97 & 2.16 & 6.8 & 0.29 & 46.11 & 21 \\
\hline SAMF7 & 60.15 & 0.32 & 6.41 & 1.68 & 0.08 & 0.97 & 14.85 & 1.11 & 1.24 & 0.05 & 13.03 & 99.91 & 56 & 58 & 3.16 & 9.4 & 0.19 & 1.12 & 20 \\
\hline SAMF9 & 57.09 & 0.41 & 7.85 & 2.48 & 0.06 & 0.59 & 15.19 & 0.79 & 1.96 & $\begin{array}{ll}5 & 0.07\end{array}$ & 13.39 & 99.88 & 62 & 69 & 2.74 & 7.3 & 0.25 & 2.5 & 19 \\
\hline SAMF16 & 63.77 & 0.39 & 8.45 & 2.04 & 0.09 & 0.89 & 10.72 & 1.1 & 2.01 & 0.05 & 10.44 & 99.95 & 59 & 63 & 2.04 & 7.5 & 0.24 & 1.84 & 22 \\
\hline SAMF19 & 65.92 & 0.42 & 8.27 & 2.29 & 0.05 & 0.49 & 10.09 & 0.63 & 2.23 & 30.07 & 9.48 & 99.94 & 65 & 72 & 1.96 & 8 & 0.27 & 3.54 & 20 \\
\hline SAMF26 & 67.68 & 0.36 & 7.69 & 2.28 & 0.09 & 1.49 & 9.09 & 0.76 & 1.44 & 0.06 & 9.03 & 99.95 & 66 & 71 & 2.01 & 8.8 & 0.19 & 1.91 & 22 \\
\hline SAMF36 & 63.45 & 0.48 & 8.07 & 2.82 & 0.08 & 1.77 & 10.49 & 1.27 & 1.16 & $5 \quad 0.06$ & 10.25 & 99.89 & 60 & 62 & 2.24 & 7.9 & 0.14 & 0.91 & 17 \\
\hline Promedio & $\begin{array}{l}62 \\
\pm 4.1\end{array}$ & $\begin{array}{c}0.4 \\
\pm 0.06\end{array}$ & $\begin{array}{l}8 \\
\pm 0.74\end{array}$ & $\begin{array}{r}2.3 \\
\pm 0.4\end{array}$ & $\begin{array}{r}0.08 \\
\pm 0.03\end{array}$ & $\begin{array}{r}0.9 \\
\pm 0.4\end{array}$ & $\begin{array}{l}12.33 \\
\pm 2.5\end{array}$ & $\begin{array}{r}0.85 \\
\pm 0.39\end{array}$ & $\begin{array}{r}1.79 \\
\pm 0.50\end{array}$ & $\begin{array}{r}0.06 \\
\pm 0.01\end{array}$ & $\begin{array}{l}11.38 \\
\pm 1.8\end{array}$ & $\begin{array}{c}99.9 \\
\pm 0.03\end{array}$ & $\begin{array}{r}63 \\
\pm 6\end{array}$ & $\begin{array}{r}70 \\
\pm 12\end{array}$ & $\begin{array}{r}2.36 \\
\pm 0.42\end{array}$ & $\begin{array}{r}7.8 \\
\pm 0.9\end{array}$ & $\begin{array}{r}0.22 \\
\pm 0.05\end{array}$ & $\begin{array}{r}1.90 \\
\pm 0.89\end{array}$ & $\begin{array}{r}21 \\
\pm 3\end{array}$ \\
\hline \multicolumn{20}{|l|}{ Lutita } \\
\hline SAMF12 & 58.45 & 0.68 & 16.53 & 6.06 & 0.04 & 1.79 & 4.15 & 0.15 & 4.71 & 0.12 & 7.26 & 99.95 & 75 & 96 & 1.06 & 3.5 & 0.28 & 31.21 & 24 \\
\hline SAMF15 & 49.87 & 0.39 & 9.89 & 2.84 & 0.14 & 2.29 & 15.35 & 0.23 & 2.85 & 0.07 & 16.07 & 99.98 & 72 & 90 & 2.44 & 5 & 0.29 & 12.62 & 25 \\
\hline SAI & 65.33 & 0.57 & 12.53 & 3.91 & & 0.99 & & 0.3 & 3.64 & 0.09 & 7.02 & 99.96 & 71 & 88 & 1.2 & 5.2 & 0.29 & 10.63 & 22 \\
\hline SAMF31 & 63.58 & 0.64 & 15.64 & 4.68 & 0.04 & 2.5 & 2.6 & 1.97 & 2.66 & 0.14 & 5.41 & 99.85 & 63 & 66 & 0.96 & 4.1 & 0.17 & 1.35 & 24 \\
\hline SAMF32 & 65.84 & 0.82 & 16.82 & 4.44 & 0.03 & 2.36 & 0.63 & 1.08 & 2.72 & 0.13 & 5.07 & 99.94 & 74 & 83 & 0.72 & 3.9 & 0.16 & 2.51 & 21 \\
\hline SAMF33 & 61.45 & 0.61 & 12.98 & 5.47 & 0.07 & 2.57 & 5.57 & 2.49 & 1.36 & 0.13 & 7.28 & 99.96 & 57 & 58 & 1.4 & 4.7 & 0.1 & 0.55 & 21 \\
\hline SAMF34 & 65.33 & 0.76 & 17.05 & 4.76 & 0.03 & 1.79 & 0.49 & 0.41 & 3.92 & 0.07 & 5.35 & 99.94 & 75 & 90 & 0.71 & 3.8 & 0.23 & 9.51 & 22 \\
\hline romedio & $\begin{array}{c}61 \\
\pm 5.3\end{array}$ & $\begin{array}{r}0.64 \\
\pm 0.13\end{array}$ & $\begin{array}{c}14 \\
\pm 2.53\end{array}$ & $\begin{aligned} & 4.59 \\
\pm & 0.9\end{aligned}$ & $\begin{array}{r}0.06 \\
\pm 0.04\end{array}$ & $\begin{array}{r}2.04 \\
\pm 0.52\end{array}$ & $\begin{array}{r}4.89 \\
+4.68\end{array}$ & $\begin{array}{r}0.95 \\
\pm 0.86\end{array}$ & $\begin{array}{c}3.12 \\
\pm 1\end{array}$ & $\begin{array}{r}0.11 \\
\pm 0.03\end{array}$ & $\begin{array}{r}7.64 \\
\pm 3.56\end{array}$ & $\begin{array}{c}99.9 \\
\pm 0.04\end{array}$ & $\begin{array}{r}70 \\
\pm 6\end{array}$ & $\begin{array}{r}82 \\
\pm 13\end{array}$ & $\begin{array}{r}1.21 \\
\pm 0.55\end{array}$ & $\begin{array}{r}4.3 \\
\pm 0.6\end{array}$ & $\begin{array}{r}0.22 \\
\pm 0.07\end{array}$ & $\begin{array}{r}9.77 \\
\pm 9.83\end{array}$ & $\begin{array}{r}23 \\
\pm 2\end{array}$ \\
\hline
\end{tabular}

0.3 y 0.9 (Cox y Lowe, 1995). En el presente estudio, los valores de la relación varían de la siguiente manera: areniscas $0.15 \pm 0.06(\mathrm{n}=20)$, limolitas $0.22 \pm 0.05(n=8)$ y lutitas $0.22 \pm 0.07(n=7)$. Estos valores sugieren que esas muestras contienen cantidades considerablemente mayores de minerales de arcilla que de minerales portadores de $\mathrm{K}$ tales como feldespatos potásicos y micas.

\section{Sección Rancho Búfalo}

Tanto las areniscas como las limolitas de esta sección tienen una alta

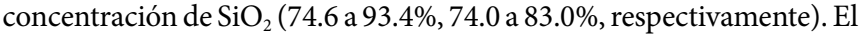
contenido de $\mathrm{Al}_{2} \mathrm{O}_{3}$ es más alto en las limolitas (9.4 a 9.9\%) que en las areniscas (3.1 a 8.9\%). También, las limolitas muestran un rango más amplio en su contenido de $\mathrm{Fe}_{2} \mathrm{O}_{3}(0.69$ a $6.53 \%)$ que las areniscas $(0.46$ a $2.14 \%)$. Por otra parte, las areniscas muestran mayores variaciones en contenido de $\mathrm{CaO}(0.21-6.91 \%)$ que las limolitas (0.37-1.43\%). Las areniscas y limolitas tienen más bajas concentraciones de $\mathrm{K}_{2} \mathrm{O}$ que de $\mathrm{Na}_{2} \mathrm{O}$ (Tabla 2). Otros elementos mayores tales como el $\mathrm{TiO}_{2}, \mathrm{MnO}$, $\mathrm{MgO}$ y $\mathrm{P}_{2} \mathrm{O}_{5}$ muestran bajas concentraciones tanto en las areniscas como en las limolitas (Tabla 2). En el diagrama de Herron (1988), Figura $4 \mathrm{~b}$, que muestra el $\log \left(\mathrm{Fe}_{2} \mathrm{O}_{3} / \mathrm{K}_{2} \mathrm{O}\right)$ vs $\mathrm{Log}\left(\mathrm{SiO}_{2} / \mathrm{Al}_{2} \mathrm{O}_{3}\right)$ la mayoría de las areniscas y limolitas caen dentro del campo de sub-litarenita, litarenita y arena-Fe. Los promedios de la relación $\mathrm{K}_{2} \mathrm{O} / \mathrm{Al}_{2} \mathrm{O}_{3}$ de las areniscas y limolitas están dentro del rango de minerales de arcilla $(0.07 \pm 0.04, \mathrm{n}=24$ y $0.04 \pm 0.03, \mathrm{n}=3$, respectivamente $)$. 


\section{DISCUSIÓN}

\section{Meteorización}

La intensidad y duración de la meteorización en las rocas sedimentarias se puede evaluar mediante la relación entre los elementos alcalinos y alcalinotérreos (Nesbitt y Young, 1982). La meteorización química de sedimentos ocurre durante la exposición de la roca fuente, el transporte en sistemas fluviales y el reciclado sedimentario, donde cada ciclo involucra meteorización química adicional (Pollack, 1961; Franzinelli y Potter, 1983; Johnsson et al., 1991; Nesbitt et al., 1996). Los efectos de la meteorización química durante el transporte en sistemas fluviales han sido estudiados por diversos autores (Pollack, 1961; Breyer y Bart, 1978; Franzinelli y Potter, 1983; Johnsson et al., 1991; Nesbitt et al., 1996) y dichos efectos pueden variar desde insignificantes hasta considerables (Pollack, 1961; Breyer y Bart, 1978; Johnsson et al, 1991; Nesbitt et al., 1996, 1997) dependiendo de la labilidad de los minerales, el clima, la distancia de transporte y el marco tectónico. Durante la meteorización, los elementos solubles como el $\mathrm{Na}$ y Ca son mayormente removidos, mientras que los elementos insolubles tales como $\mathrm{Al}$ son generalmente fijados en el perfil de meteorización (Nesbitt et al., 1980). Tales cambios químicos son transferidos al registro sedimentario (e.g., Nesbitt y Young, 1982; Wronkiewicz y Condie, 1987), proporcionando por lo tanto una herramienta útil para el monitoreo de las condiciones de meteorización del área fuente.

Nesbitt et al. (1997) propusieron un perfil de meteorización idealizada dividido en cuatro zonas mineralógicas. La zona IV, inmediatamente por encima de la roca madre, es la menos alterada, con abundancia de fragmentos de roca, cuarzo y feldespatos y ausencia de minerales de arcilla. La zona III se caracteriza por la abundancia de granos de cuarzo y feldespatos, sin embargo se tienen, cantidades apreciables de fragmentos de roca y pequeñas cantidades de minerales de arcilla complejos (esmectita, vermiculita e ilita). La zona II, consiste en una abundancia de cuarzo y feldespatos, abundante mineral de arcilla y cantidades menores o ausencia de fragmentos de roca. Por último, la Zona I, la cual es la zona más intensamente meteorizada contiene abundante cuarzo, en segundo lugar minerales de arcilla y en tercero los productos de alteración (oxihidróxidos de $\mathrm{Al}, \mathrm{Fe}$ y Ti).

Diversos autores han propuesto varios tipos de índices de meteorización (Nesbitt y Young, 1982; Harnois, 1988), que son generalmente usados por varios investigadores (Madhavaraju y Ramasamy, 2002; Armstrong-Altrin et al., 2004; Nagarajan et al., 2007; Bhuiyan et al., 2011; Sun et al., 2012; Zaid, 2012; Madhavaraju, 2015; Ramachandran et al., 2016). El índice químico de alteración (CIA: Nesbitt y Young, 1982) es ampliamente usado en los estudios geoquímicos sedimentarios para evaluar el grado de meteorización en el área fuente. Este valor se calcula utilizando las proporciones moleculares de la siguiente fórmula: $\mathrm{CIA}=\left[\mathrm{Al}_{2} \mathrm{O}_{3} /\left(\mathrm{Al}_{2} \mathrm{O}_{3}+\mathrm{CaO}^{*}+\mathrm{Na}_{2} \mathrm{O}+\mathrm{K}_{2} \mathrm{O}\right)\right] \times 100$ (Nesbitt y Young, 1982), donde $\mathrm{CaO}^{\star}$ representa la cantidad de óxido de calcio en los minerales silicatados solamente. En el presente estudio, hemos seguido el método propuesto por McLennan (1993) para calcular el CaO ${ }^{\star}$, en el cual los valores de $\mathrm{CaO}$ son aceptados solamente si $\mathrm{CaO}<\mathrm{Na}_{2} \mathrm{O}$ y, cuando $\mathrm{CaO}>\mathrm{Na}_{2} \mathrm{O}$, se asume que el contenido de $\mathrm{CaO}$ equivale al de $\mathrm{Na}_{2} \mathrm{O}$ y por lo tanto se sustituye con el mismo.

Las areniscas de la columna de la Sierra Anibacachi muestran las variaciones más grandes en los valores CIA (40-88), mientras que las limolitas y lutitas varían de 56 a 75 y 57 a 75, respectivamente (Tabla 1). Las rocas no meteorizadas exhiben valores CIA de 50 (Nesbitt y Young, 1982) y el valor CIA para el rango promedio de las lutitas es de 70-75 (Taylor y McLennan, 1985). Por otra parte, las rocas fuente intensamente meteorizadas muestran valores de 100 para CIA y la composición mineral tiende hacia caolinita o gibbsita (Nesbitt y Young, 1982). El valor PIA (Índice de alteración de plagioclasas) es otro índice para inferir la intensidad de meteorización química de las rocas sedimentarias y puede ser calculado de la siguiente manera: $\mathrm{PIA}=\left[\left(\mathrm{Al}_{2} \mathrm{O}_{3}-\mathrm{K}_{2} \mathrm{O}\right) /\left(\mathrm{Al}_{2} \mathrm{O}_{3}+\mathrm{CaO}^{*}+\mathrm{Na}_{2} \mathrm{O}-\mathrm{K}_{2} \mathrm{O}\right)\right] \times 100$, utilizando la proporción molecular de cada óxido mencionado (Fedo et al., 1995). Los valores PIA de las muestras de areniscas, limolitas y lutitas estu-

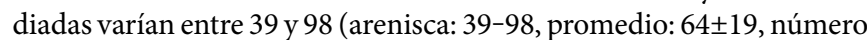
de muestras, $n=20$; limolita: 58-97, 70 \pm 12 , $n=8$; lutita: 58-96, 82 \pm 13 , $\mathrm{n}=7$; Tabla 1). Las amplias variaciones observadas en los valores CIA y PIA de las areniscas indican una baja a intensa meteorización en el área fuente, mientras que los valores CIA para las limolitas y lutitas muestran una intensidad moderada de meteorización química en el área fuente. Los valores PIA de las areniscas, limolitas y lutitas son consistentes con los valores CIA.

Por otro lado, para las rocas de la columna de Rancho Búfalo los valores CIA varían de 44 a 91 (arenisca: 44-78; excepto una muestra (RBMF22) la cual presenta un alto valor CIA de 91; limolitas: 50-69; Tabla 2). De igual manera, las areniscas muestran mayores variaciones en sus valores de PIA (44-96, 60 $\pm 14, \mathrm{n}=24)$ que las limolitas (50-71, $59 \pm 11, n=3$; Tabla 2), lo que sugiere que el área fuente de la cual se derivaron las areniscas fue meteorizada con una intensidad baja a intensa, mientras que las limolitas recibieron sedimentos de una roca fuente afectada con un bajo a moderado grado de meteorización química.

Además, un diagrama ternario $\mathrm{Al}_{2} \mathrm{O}_{3}-\mathrm{CaO}^{*}+\mathrm{Na}_{2} \mathrm{O}-\mathrm{K}_{2} \mathrm{O}(\mathrm{A}-$ $\mathrm{CN}-\mathrm{K})$ es una herramienta importante con la cual examinar la tendencia de meteorización de las rocas fuente cuando la corteza superior está dominada por rocas ricas en plagioclasas y feldespatos-K y sus productos de la meteorización (Nesbitt y Young, 1984, 1989; Fedo et al., 1995). Durante la etapa inicial de meteorización, los sedimentos derivados de las rocas ígneas se representan principalmente a lo largo de la línea $\mathrm{A}-\mathrm{CN}$ porque el $\mathrm{Na}_{2} \mathrm{O}$ y $\mathrm{CaO}$ son lixiviados de la plagioclasa disuelta anteriormente. La destrucción de la plagioclasa ocurre cuando se tiene un incremento en la intensidad de meteorización de las rocas del área fuente, que conduce a la pérdida significativa de Ca y $\mathrm{Na}$ de la plagioclasa y los sedimentos resultantes se representan principalmente paralelos al eje A-K. En el diagrama A-CN-K (Figura 5a), la mayoría de las areniscas y limolitas así como algunas lutitas de la sección Sierra Anibacachi siguen una tendencia lineal (línea discontinua en la Figura 5a) paralela al plano A-CN, mientras que la mayoría de las lutitas y escasas arenisca se grafican paralelas al plano A-K y sólo algunas muestras de arenisca se aproximan hacia "A" (Figura 5a). La mayoría de las areniscas y de las limolitas forman un grupo definido, mientras la mayoría de las lutitas constituyen un grupo reducido y escasas muestras de arenisca forman otro grupo. Una gran parte de nuestras muestras de arenisca aparecen cercanas a la línea de unión de feldespatos, sugiriendo que las Zonas III y IV contribuyeron significantemente a los sedimentos (vea la zona de clasificación en Nesbitt et al., 1997). Pocas areniscas caen cercanas al límite A-K, lo que indica una remoción de plagioclasa producto de la meteorización química y un enriquecimiento residual de cuarzo. Lo anterior sugiere que esas areniscas fueron principalmente derivadas de la Zona II. Dos muestras de lutita se representan ligeramente alejadas de la línea de unión de feldespatos indicando que prácticamente ha ocurrido una mínima meteorización química y que las muestras de lutita se componen principalmente de rocas de basamento trituradas en lugar de minerales de arcilla aluminosos. El resto de las muestras se representan como un grupo y se localizan a lo largo de la línea $\mathrm{A}-\mathrm{K}$, sugiriendo que las lutitas recibieron sedimentos principalmente de las Zonas III y IV. Esto además, apoya la idea de que las areniscas fueron sometidas a una intensidad de meteorización química de baja a intensa en el área fuente, mientras que las limolitas y lutitas recibieron los sedimentos de rocas fuente meteorizadas con una intensidad moderada. 
Tabla 2. Datos de óxidos mayores (wt\%) para areniscas y limolitas de la Formación Morita en la sección Rancho Búfalo.

\begin{tabular}{|c|c|c|c|c|c|c|c|c|c|c|c|c|c|c|c|c|c|c|c|}
\hline $\begin{array}{l}\text { Muestra } \\
\text { Núm. }\end{array}$ & $\mathrm{iO}_{2}$ & $\mathrm{iO}_{2}$ & $\mathrm{Al}_{2} \mathrm{O}_{3}$ & $\mathrm{Fe}_{2} \mathrm{O}_{3}$ & MnO & $\mathrm{MgO}$ & $\mathrm{CaO}$ & $\mathrm{Na}_{2} \mathrm{O}$ & $\mathrm{K}_{2} \mathrm{O}$ & $\mathbf{P}_{2} \mathbf{O}_{5}$ & PC & Suma & CIA & PIA & ICV & $\begin{array}{l}\mathrm{SiO}_{2} / \\
\mathrm{Al}_{2} \mathrm{O}_{3}\end{array}$ & $\begin{array}{c}\mathrm{K}_{2} \mathrm{O} / \\
\mathrm{Al}^{2} \mathrm{O}_{3}\end{array}$ & $\begin{array}{l}\mathrm{K}_{2} \mathrm{O} / \\
\mathrm{Na}_{2} \mathrm{O}\end{array}$ & $\begin{array}{c}\mathrm{Al}_{2} \mathbf{O}_{3} / \\
\mathrm{TiO}_{2}\end{array}$ \\
\hline \multicolumn{20}{|l|}{ Arenisca } \\
\hline $3 N$ & 74.55 & 0.28 & 57 & 2.14 & 0.09 & 0.62 & 4.73 & 2.9 & 1.12 & 0.05 & 5.1 & 100.14 & 44 & 44 & 38 & 8.7 & 0.13 & 0.39 & 31 \\
\hline BMF3 & .01 & 0.13 & 19 & 0.99 & 6 & & 3 & 8 & 9 & & 1.95 & 0.16 & 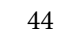 & 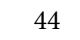 & 97 & 11.7 & 0.07 & .15 & 54 \\
\hline RBMF4 & 83.24 & 0.36 & 8.77 & 1.7 & 0.02 & 0.35 & 0.31 & 4 & 0.33 & 0.05 & 0.9 & 100.02 & 54 & 54 & 81 & 9.5 & 0.04 & 0.08 & 25 \\
\hline 3MF5 & 85.55 & 0. & 7.29 & 1. & 0.02 & 0.33 & 0.4 & 3.14 & 0.48 & 0.02 & 1.05 & 100.02 & 5 & 5 & .84 & 11.7 & 0.07 & 0.15 & 39 \\
\hline 66 & 39 & 0 & 4 & & 3 & & & & 6 & & 6 & & & & 76 & 0.5 & 4 & 11 & 37 \\
\hline BMF8 & .71 & 0.13 & 8.92 & 1.56 & 0.04 & 0.52 & 0.76 & 2.85 & 0.51 & & 2.14 & 00.16 & ני & 8 & 71 & 9.3 & 0.06 & 18 & 70 \\
\hline BMF10 & 79.56 & 0.12 & 7.3 & 0.85 & 0.15 & 0.19 & 4.09 & 4.22 & & & 3.57 & 10 & & & 1.32 & 10.9 & & 0.01 & 59 \\
\hline BMF12 & 85.69 & 0.04 & 7.65 & 17 & 0.01 & 0.55 & 0.35 & 03 & 0.55 & 0 & 1.9 & 9.96 & 63 & 6 & 61 & 1.2 & .07 & .27 & 212 \\
\hline BMF13 & 83.92 & 0.22 & 8.18 & 1. & 0.02 & 4 & 0.28 & .94 & 0.81 & & 1.41 & 100.15 & 5 & & 81 & 0.3 & & .28 & 37 \\
\hline RBMF14 & 90.15 & 0.09 & 5.19 & & 0.01 & 0. & 0.31 & 1 & 0. & & 1.2 & 10 & 3 & 57 & 69 & 17.4 & 0.06 & 0.16 & 60 \\
\hline BMF15 & 87.53 & 0.18 & 6.5 & 0.81 & 1 & 0.72 & 0.4 & 1.4 & 0. & & 2.14 & 100.17 & 6 & & 61 & 13.5 & 66 & 0.26 & 37 \\
\hline RBMF16 & 85.69 & 0. & 5.65 & 1.01 & 0.03 & 0.64 & 1.82 & 11 & 0.73 & 0 & 2.85 & 99.84 & 5 & & 99 & 15.2 & 13 & 0.62 & 27 \\
\hline RBMF17 & 90.85 & 0.17 & 4.73 & 0.68 & 0.01 & 0.4 & 0.24 & 1.18 & 0.21 & & 1.42 & 100.07 & 65 & 6 & 0.61 & 19.2 & 0.04 & 0.18 & 29 \\
\hline RBMF18 & 88.52 & 0.02 & 3.15 & 0.46 & 0.06 & 0.35 & 3.1 & 0.97 & 0.21 & 0. & 3.14 & 100 & 4 & 48 & 1.64 & 28.1 & 0.07 & 0.22 & 175 \\
\hline RBMF19 & 80.53 & 0.18 & 3.96 & 0.75 & 0.08 & 0.5 & 6.43 & & 0.41 & 0.04 & 5.92 & 100.03 & A & 47 & 2.42 & 20.3 & 0.1 & 0.35 & 23 \\
\hline RBMF20 & 86.72 & 0.19 & 4.11 & 0.84 & 0.05 & 0.57 & 2.94 & 0.93 & 0.4 & 0.04 & 3.34 & 100.12 & 54 & 55 & 1.44 & 21.1 & 0.1 & 0.43 & 22 \\
\hline RBMF21 & 84.16 & 0.3 & 3.77 & & 0.06 & 0.37 & 4.87 & 0.09 & 0.71 & 0. & 5.26 & 100 & 78 & 91 & 1.82 & 22.3 & 0.19 & 8.24 & 12 \\
\hline RBMF22 & 93.38 & 0.17 & 3.47 & 0.73 & 0.04 & 0.35 & 0.21 & 0.05 & 0.16 & 0.0 & 1.49 & 100.09 & 91 & 96 & 0.49 & 26.9 & 0.05 & 3.49 & 21 \\
\hline RBMF23 & 76 & 0.27 & 5.5 & 1.28 & 0.09 & 1.24 & 6.91 & 0.82 & 0.68 & 0.05 & 7.3 & 100.14 & 62 & 64 & 2.05 & 13.8 & 0.12 & 0.83 & 20 \\
\hline RBMF24 & 79.12 & 0.43 & 4.24 & 0.96 & 0.06 & 0.72 & 6.79 & 0.47 & 0.38 & 0.05 & 6.82 & 100.04 & 68 & 71 & 2.31 & 18.7 & 0.09 & 0 . & 10 \\
\hline RBMF25 & 81.32 & 0.28 & 5.09 & 1.91 & 0.06 & 1.75 & 3.8 & & 0. & 0.04 & 4.69 & 100.11 & 61 & 62 & 1.77 & 16 & 0.06 & 0.33 & 18 \\
\hline BMF26 & 80.65 & 0.44 & 4.84 & 16 & 0.07 & 1.76 & 5.19 & 0.76 & 0.18 & 0.04 & 56 & 101.12 & 64 & 6 & 2.07 & 16.7 & 0.04 & 0.24 & 11 \\
\hline RBMF28 & 89.14 & 0.24 & 3.74 & 1.18 & 0.08 & 0.67 & 1.71 & 0.01 & 0.15 & 0.06 & 3.17 & 100.14 & 53 & 53 & 1.08 & 23.9 & 0.04 & 15.4 & 16 \\
\hline RBMF29 & 82.06 & 0.25 & 3.14 & 0.72 & 0.04 & 0.59 & 6.34 & 0.36 & 0.1 & 0.03 & 6.47 & 100.15 & 70 & 71 & 2.7 & 26.2 & 0.05 & 0.42 & 12 \\
\hline Promedio & $\begin{array}{c}84.1 \\
\pm 0.11\end{array}$ & $\begin{array}{r}0.21 \\
\pm 0.11\end{array}$ & $\begin{array}{r}5.7 \\
\pm 1.9\end{array}$ & $\begin{array}{r}1.11 \\
\pm 0.48\end{array}$ & $\begin{array}{r}0.05 \\
\pm 0.03\end{array}$ & $\begin{array}{l}0.62 \\
\pm 0.4\end{array}$ & $\begin{array}{r}2.69 \\
\pm 2.43\end{array}$ & $\begin{array}{r}1.70 \\
\pm 1.29\end{array}$ & $\begin{array}{r}0.42 \\
\pm 0.25\end{array}$ & & & & $\begin{array}{r}59 \\
\pm 11\end{array}$ & $\begin{array}{r}60 \\
+14\end{array}$ & $\begin{array}{r}1.29 \\
\pm 0.66\end{array}$ & $\begin{array}{l}16.4 \\
\pm 6\end{array}$ & $\begin{array}{r}0.07 \\
\pm 0.04\end{array}$ & $\begin{array}{r}0.31 \\
\pm 0.22\end{array}$ & $\begin{array}{r}30 \\
\pm 17\end{array}$ \\
\hline \multicolumn{20}{|l|}{ Limolita } \\
\hline & 78.54 & 0.33 & 9.78 & 2.53 & 0.05 & 1.02 & 1.45 & 2.65 & 0 . & 0 & 3.03 & 100.1 & 56 & 56 & 89 & 8 & 0.07 & 0.26 & 30 \\
\hline & 83.0 & 0.17 & & 0. & 0.0 & 0.2 & 0.37 & & & & 0.83 & & 50 & 50 & 0.73 & 8.9 & 0.01 & 0.01 & 55 \\
\hline RBMF11 & 74 & 0.44 & 9.86 & 6.53 & 0.1 & 2.09 & 1.29 & 1.17 & 0.54 & 0.03 & 4.04 & 100.09 & 69 & 71 & 1.23 & 7.5 & 0.05 & 0.46 & 22 \\
\hline Promedio & $\begin{array}{r}78.5 \\
\pm 4.5\end{array}$ & $\begin{array}{r}0.31 \\
+0.14\end{array}$ & $\begin{array}{r}9.6 \\
\pm 0.2\end{array}$ & $\begin{array}{l}3.25 \\
+2.9\end{array}$ & $\begin{array}{r}0.05 \\
\pm 0.04\end{array}$ & $\begin{array}{r}1.12 \\
\pm 0.93\end{array}$ & $\begin{array}{r}1.03 \\
\pm 0.58\end{array}$ & $\begin{aligned} & 3.05 \\
\pm & 2.1\end{aligned}$ & $\begin{aligned} & 0.43 \\
= & 0.33\end{aligned}$ & $\begin{aligned} & 0.03 \\
= & 0.0\end{aligned}$ & $\begin{array}{r}2.63 \\
=1.64\end{array}$ & $\begin{array}{l}100 \\
\pm 0.01\end{array}$ & $\begin{array}{r}58 \\
\pm 10\end{array}$ & $\begin{array}{r}59 \\
\pm 11\end{array}$ & $\begin{array}{r}0.95 \\
+0.26\end{array}$ & $\begin{array}{r}8.1 \\
\pm 0.7\end{array}$ & $\begin{array}{r}0.04 \\
\pm 0.03\end{array}$ & $\begin{array}{r}0.24 \\
\pm 0.23\end{array}$ & $\begin{array}{l}36 \\
17\end{array}$ \\
\hline
\end{tabular}

De igual manera, en el diagrama A-CN-K, la mayoría de las areniscas y limolitas de la sección Rancho Búfalo forman una tendencia lineal (línea discontinua) paralela al plano A-CN, en el cual una muestra se aproxima al vértice "A" (Figura 5b). Muchas areniscas y sólo dos limolitas se ubican cerca de la línea de unión de feldespatos, mientras que algunas areniscas y solamente una limolita se representan alejadas de la línea de unión de los feldespatos, lo que indica que esas areniscas y limolitas son derivadas principalmente de las Zonas III y IV del perfil de meteorización en la región de origen (e.g., Nesbitt et al., 1997). Una arenisca se representa cerca al vértice "A", indicando que tiene aporte de la Zona II. Esto sugiere que esas areniscas y limolitas recibieron sedimentos de rocas fuente afectadas por un grado de meteorización química de bajo a moderado (excepto la muestra RBMF22, la cual presenta un intenso grado de meteorización química).

El diagrama ternario A-CN-K puede ser usado para ilustrar los valores CIA gráficamente, para inferir la composición de las rocas del área fuente, la intensidad del metasomatismo-K postdepositacional y para evaluar el rol de meteorización estática y no estática (Nesbitt y Young, 1984; Fedo et al., 1995; Nesbitt et al., 1996, 1997). En el presente estudio, las rocas siliciclásticas de ambas secciones muestran valores CIA bastante variables y también exhiben una importante dispersión a lo largo de la tendencia de meteorización proyectada. Tal meteorización no uniforme es típica de un estado de condiciones no estáticas, lo que indica un tectonismo activo que permite la erosión de todas las zonas dentro del perfil de meteorización desarrolladas sobre la roca fuente (e.g., Nesbitt et al., 1997).

La intensidad de la meteorización química puede ser inferida utilizando el Índice Químico de Variabilidad (ICV por sus siglas en inglés; Cox et al., 1995). Durante la meteorización química, el contenido de alúmina se incrementa y simultáneamente el contenido de materiales ferromagnesianos disminuye. Por lo tanto, estos dos parámetros proporcionan la información respecto a la madurez de las rocas sedimentarias. La madurez de las rocas sedimentarias se calcula utilizando los valores ICV $\left(\mathrm{ICV}=\left(\mathrm{CaO}+\mathrm{Na}_{2} \mathrm{O}+\mathrm{Fe}_{2} \mathrm{O}_{3}+\mathrm{MgO}+\mathrm{MnO}\right.\right.$ $\left.\left.+\mathrm{TiO}_{2}\right) / \mathrm{Al}_{2} \mathrm{O}_{3}\right)(\mathrm{Cox}$ et al., 1995). El intervalo y promedio de lo valores ICV para las areniscas, limolitas y lutitas de la Sierra Anibacachi varían significantemente $(0.24-3.73,1.64 \pm 1.04, \mathrm{n}=20 ; 1.96-3.16,2.36 \pm 0.42$, $\mathrm{n}=8 ; 0.71-2.41,1.21 \pm 0.55, \mathrm{n}=7$; respectivamente). En cambio, los valores ICV para las areniscas y limolitas de la sección Rancho Búfalo tienen un rango entre $0.49-2.70(1.29 \pm 0.66, \mathrm{n}=24)$ y entre $0.73-1.23$ $(0.95 \pm 0.26, \mathrm{n}=3)$, respectivamente. Las rocas siliciclásticas de estas dos secciones muestran grandes variaciones en los valores ICV, indicando que sus rocas siliciclásticas son composicionalmente de carácter inmaduro a maduro. Las rocas sedimentarias con tales variaciones amplias en valores ICV se encuentran principalmente en los campos tectónicamente activos (Cox et al., 1995), donde los sedimentos 

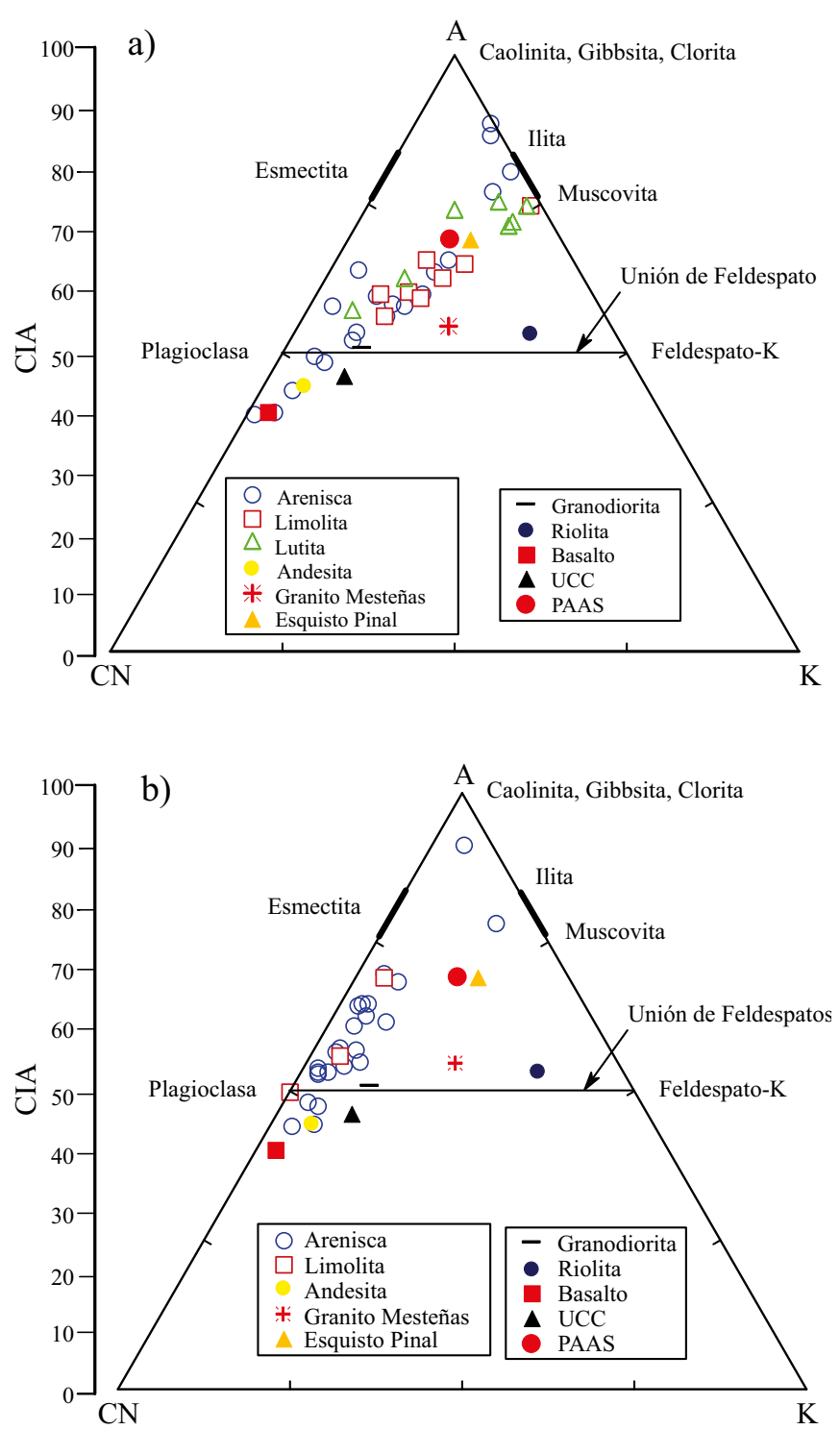

Figura 5. a) Diagrama A-CN-K mostrando la tendencia de meteorización de areniscas, limolitas y lutitas de la sección Sierra Anibacachi, y b) Diagrama A-CN-K mostrando la línea de meteorización de areniscas y limolitas de la sección Rancho Búfalo de la Formación Morita (Nesbitt y Young, 1982) A: $\mathrm{Al}_{2} \mathrm{O}_{3} ; \mathrm{CN}: \mathrm{CaO}^{*}+\mathrm{Na}_{2} \mathrm{O} ; \mathrm{K}: \mathrm{K}_{2} \mathrm{O}$ (proporciones moleculares). Esquisto Pinal y Granito Mestañas (este estudio), Basalto y datos de UCC de Condie, 1993.

pueden ser derivados de varias zonas que contienen sedimentos inmaduros a maduros. Eso no permitió que los sedimentos perdieran elementos solubles y que los minerales primarios, como feldespato, se transformaran en arcillas ricas en aluminio. Por lo tanto, los sedimentos resultantes tienen valores ICV muy altos (e.g., Mondal et al., 2012). Los datos presentados aquí sugieren que las rocas sedimentarias con mayores valores ICV derivaron principalmente de la Zona IV del perfil de intemperismo de la región fuente.

\section{Marco tectónico}

La petrografía y geoquímica de las rocas clásticas se ha utilizado comúnmente para discriminar los ambientes tectónicos de cuencas antiguas (Dickinson y Suczek, 1979; Maynard et al., 1982; Bhatia, 1983; Dickinson et al., 1983; Roser y Korsch, 1985, 1986, 1988; Taylor y McLennan, 1985; Bhatia y Crook, 1986; McLennan y Taylor, 1991;
Girty et al., 1993; Spalletti et al., 1991, 1992; McLennan et al., 1993; Murray, 1994). Sin embargo, numerosos investigadores han expresado serias dudas sobre la posibilidad de discriminar los marcos tectónicos a partir del uso de datos geoquímicos (e.g., Ryan y Williams, 2007; Pe-Piper et al., 2008) debido a los diversos factores considerados, tales como la litología, ambiente tectónico, clima, relieve y la pendiente del área fuente.

Bhatia (1983) y Roser y Korsh (1986) han propuesto diversos diagramas como $\mathrm{TiO}_{2}$ vs $\mathrm{Fe}_{2} \mathrm{O}_{3}+\mathrm{MgO} \mathrm{Al} \mathrm{A}_{3} / \mathrm{SiO}_{2}$ vs $\mathrm{Fe}_{2} \mathrm{O}_{3}+\mathrm{MgO}$ y $\mathrm{K}_{2} \mathrm{O} / \mathrm{Na}_{2} \mathrm{O}$ vs $\mathrm{SiO}_{2}$ para identificar los ambientes tectónicos de cuencas desconocidas. Esos diagramas son extensamente usados, pero no integran análisis estadísticos lógicos de datos composicionales (Thomas y Aitchison, 2005; Agrawal y Verma, 2007; Verma, 2010, 2012; Verma y Armstrong-Altrin, 2013) y diversos autores han expresado preocupación sobre la posibilidad de discriminar marcos tectónicos a partir de los datos geoquímicos (e.g., Ryan y Williams, 2007; Pe-Piper et al., 2008; von Eynatten y Dunkl, 2012). Los diagramas de discriminación de marco tectónico propuestos por Bhatia (1983) y Roser y Korsch (1986) fueron evaluados por Armstrong-Altrin y Verma (2005) y se obtuvo que la relación de éxito para los diagramas es muy bajo. Varios autores han expresado la desventaja de esos diagramas discriminatorios tradicionales para rocas silisiclásticas (e.g., Weltje, 2006, 2012; Caja et al., 2007; Borges et al., 2008; Armstrong-Altrin, 2009; Blanco et al., 2011; Guo et al., 2011; Caracciolo et al., 2012; Ghosh et al., 2012; Zaid, 2012).

Verma y Armstrong-Altrin (2013) propusieron dos nuevos diagramas de función discriminante basados en contenidos de óxidos mayores de sedimentos siliciclásticos para discriminar ambientes tectónicos de arcos de islas o continentales, rift continental, y en ambientes de colisión. Estos diagramas incluyen la discriminación tectónica basada en rocas con alto sílice $\left[\left(\mathrm{SiO}_{2}\right)_{\mathrm{adj}}=63 \%-95 \%\right]$ y bajo sílice $\left[\left(\mathrm{SiO}_{2}\right)_{\text {adj }}=35 \%-63 \%\right]$ y fueron elaborados con base en datos publicados de rocas siliciclásticas del Neógeno-Cuaternario de ambientes tectónicos conocidos, aplicando la conversión a $\log _{\mathrm{e}}$ de los 10 elementos mayores utilizando $\mathrm{SiO}_{2}$ como el común denominador, y el análisis discriminante lineal de estas conversiones. Los valores para $\left(\mathrm{SiO}_{2}\right)$ adj se obtienen al recalcular el contenido de $\mathrm{SiO}_{2}$ cuando al total de la muestra se le resta el valor de LOI.

En el presente estudio, las rocas siliciclásticas de la Sierra Anibacahi y Rancho Búfalo muestran valores mayores a $63 \%$ de $\mathrm{SiO}_{2 \text { adj }}$. Por lo tanto, se usaron los diagramas de función discriminante basados en alto sílice con el fin de identificar los ambientes tectónicos de la Formación Morita. En este diagrama (Verma y Armstrong-Altrin, 2013) (Figura 6a), la mayoría de las areniscas, limolitas y lutitas de la Sierra Anibacachi se encuentran en el campo de colisión aunque algunas areniscas caen en los campos de arco y rift y otras de limolitas caen en la campo de rift (Figura 6a). Igualmente, para las areniscas y limolitas de la sección Rancho Búfalo se utiliza el diagrama de función discriminante basado en alto sílice y la mayoría de ellas se grafican en el campo de arco (Figura 6b). Sin embargo, algunas areniscas caen dentro del campo de colisión.

Los resultados obtenidos de los diagramas de función discriminante propuestos por Verma y Armstrong-Altrin (2013) son consistentes con la evolución tectónica regional del área de estudio. La mayoría de las muestras de la Sierra Anibacachi se representan en el campo de colisión, sin embargo, algunas muestras se grafican en los campos de arco y rift. Por otra parte, la mayoría de las muestras de la sección Rancho Búfalo se representan en el campo de arco y unas pocas muestras en el campo de colisión. Esto sugiere que las rocas siliciclásticas colectadas de la Sierra Anibacachi y Rancho Búfalo recibieron sedimentos en varias proporciones de diferentes marcos tectónicos.

Como se mencionó anteriormente, la mayoría de las muestras de la Sierra Anibacachi grafican en el campo de colisión, cuyos sedimentos 

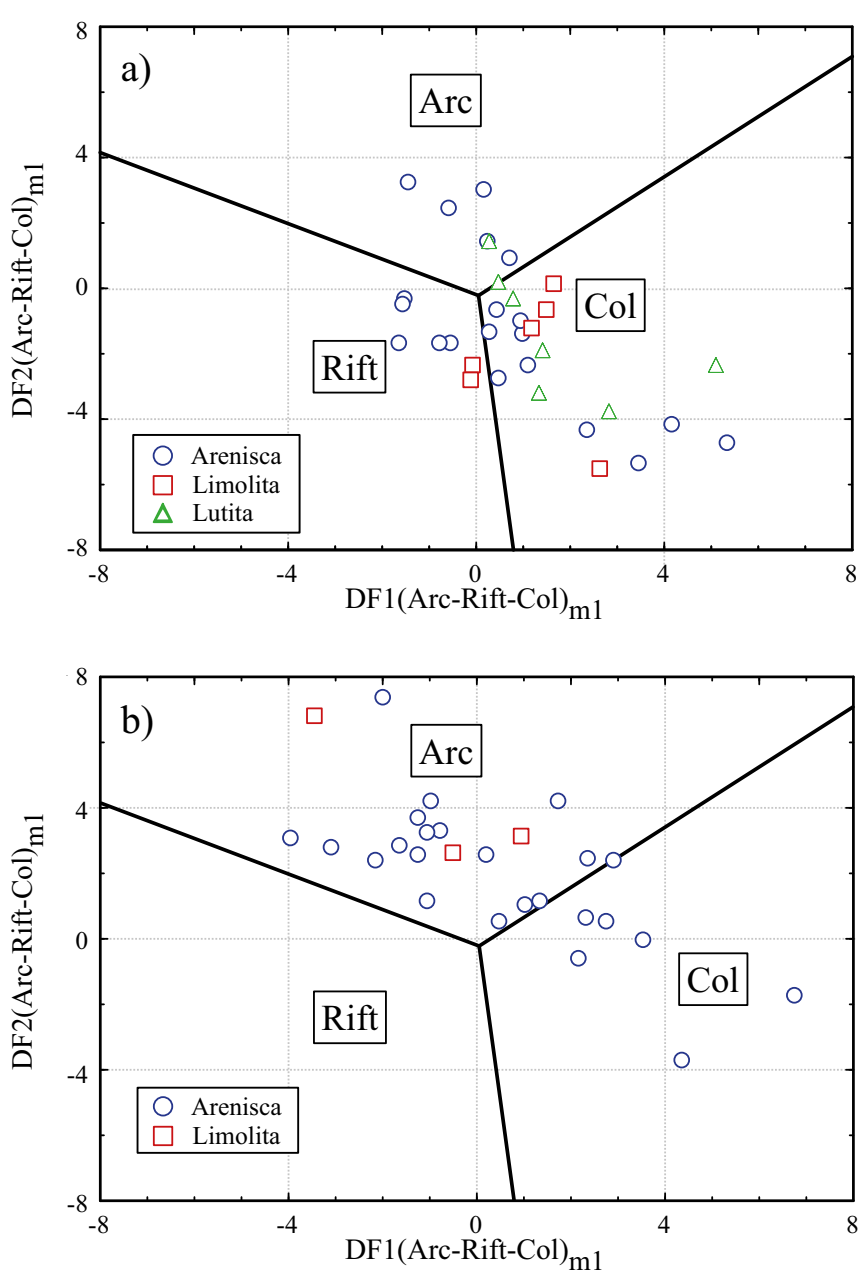

Figura 6. a) Diagrama discriminante de función multidimensional para rocas clásticas de alto sílice (areniscas, limolitas y lutitas) de la Sección Sierra Anibacachi, y b) Diagrama discriminante de función multidimensional para rocas clásticas de alto sílice (areniscas y limolitas) de la Sección Rancho Búfalo (Verma and Armstrong-Altrin, 2013). El subíndice ${ }_{\mathrm{m} 1}$ en DF1 y DF2 representa el diagrama de alto sílice basado en relaciones loge de elementos mayores. Las ecuaciones de función discriminatoria son $\mathrm{DF} 1_{(\mathrm{Arc}-\mathrm{Ritt}-\mathrm{Col}) \mathrm{ml}}=\left(-0.263 \times \operatorname{In}\left(\mathrm{TiO}_{2} /\right.\right.$ $\left.\left.\mathrm{SiO}_{2}\right)_{\text {adj }}\right)+\left(0.604 \times \mathrm{In}\left(\mathrm{Al}_{2} \mathrm{O}_{3} / \mathrm{SiO}_{2}\right)_{\text {adj }}\right)+\left(-1.725 \times \mathrm{In}\left(\mathrm{Fe}_{2} \mathrm{O}_{3}{ }^{\mathrm{t}} / \mathrm{SiO}_{2}\right)_{\text {adj }}\right)+(0.660 \times$ $\left.\mathrm{In}\left(\mathrm{MnO} / \mathrm{SiO}_{2}\right)_{\mathrm{adj}}\right)+\left(2.191 \times \mathrm{In}\left(\mathrm{MgO} / \mathrm{SiO}_{2}\right)_{\mathrm{adj}}\right)+\left(0.144 \times \mathrm{In}\left(\mathrm{CaO} / \mathrm{SiO}_{2}\right)_{\mathrm{adj}}\right)+$ $\left(-1.304 \times \operatorname{In}\left(\mathrm{Na}_{2} \mathrm{O} / \mathrm{SiO}_{2}\right)_{\text {adj }}\right)+\left(0.054 \times \operatorname{In}\left(\mathrm{K}_{2} \mathrm{O} / \mathrm{SiO}_{2}\right)_{\text {adj }}\right)+\left(-0.330 \times \operatorname{In}\left(\mathrm{P}_{2} \mathrm{O}_{5} /\right.\right.$ $\left.\left.\mathrm{SiO}_{2}\right)_{\text {adj }}\right)+1.588$. DF2 ${ }_{\text {(Arc-Rift-Col)ml }}=\left(-1.196 \times \mathrm{In}\left(\mathrm{TiO}_{2} / \mathrm{SiO}_{2}\right)_{\text {adj }}\right)+(1.604 \times$ $\left.\operatorname{In}\left(\mathrm{Al}_{2} \mathrm{O}_{3} / \mathrm{SiO}_{2}\right)_{\mathrm{adj}}\right)+\left(0.303 \times \operatorname{In}\left(\mathrm{Fe}_{2} \mathrm{O}_{3}{ }^{\mathrm{t}} / \mathrm{SiO}_{2}\right)_{\text {adj }}\right)+\left(0.436 \times \operatorname{In}\left(\mathrm{MnO} / \mathrm{SiO}_{2}\right)_{\text {adj }}\right)$ $+\left(0.838 \times \operatorname{In}\left(\mathrm{MgO} / \mathrm{SiO}_{2}\right)_{\text {adj }}\right)+\left(-0.407 \times \operatorname{In}\left(\mathrm{CaO} / \mathrm{SiO}_{2}\right)_{\text {adj }}\right)+\left(1.021 \times \operatorname{In}\left(\mathrm{Na}_{2} \mathrm{O} /\right.\right.$ $\left.\left.\mathrm{SiO}_{2}\right)_{\text {adj }}\right)+\left(-1.706 \times \operatorname{In}\left(\mathrm{K}_{2} \mathrm{O} / \mathrm{SiO}_{2}\right)_{\text {adj }}\right)+\left(-0.126 \times \operatorname{In}\left(\mathrm{P}_{2} \mathrm{O}_{5} / \mathrm{SiO}_{2}\right)_{\mathrm{adj}}\right)-1.068$.

fueron probablemente derivados de afloramientos del Esquisto Pinal Precámbrico (Condie y DeMalas, 1985) e intrusivos asociados. Los granitoides Permo-Triásicos de origen de arco magmático continental están expuestos en las áreas de Sierra Pinta, Sierra Los Tanques y Sierra San Francisco (Arvizu et al., 2009; Arvizu, 2012). Las rocas Proterozoicas formadas en un marco de arco continental están bien expuestas en el Bloque Caborca (Iriondo et al., 2004) y pueden ser consideradas como una de las rocas fuentes de las Formación Morita. Además, algunas muestras de la Sierra Anibacachi grafican en el campo de rift (Figura 6a) y sus sedimentos probablemente fueron derivados de las rocas ígneas que en el norte de Sonora son parte del arco mágmatico continental de edad Jurásica (Anderson y Silver, 2005; RodríguezCastañeda y Anderson, 2011; Lawton y Molina-Garza, 2014).

\section{CONCLUSIONES}

Se analizaron óxidos mayores de muestras de areniscas, limolitas y lutitas de la Formación Morita para inferir las condiciones de meteorización y ambientes tectónicos de las rocas de origen. En el diagrama de Herron (1988) $\log \left(\mathrm{Fe}_{2} \mathrm{O}_{3} / \mathrm{K}_{2} \mathrm{O}\right)$ vs $\mathrm{Log}\left(\mathrm{SiO}_{2} / \mathrm{Al}_{2} \mathrm{O}_{3}\right)$, las rocas siliciclásticas se ubican en los campos de sub-litarenita, litarenita, sub-arcosa y arenas-Fe. Las areniscas de la Sierra Anibacachi indican valores de CIA y PIA de una meteorización química de baja a intensa del área de aporte, mientras que para limolitas y lutitas los valores de CIA muestran una meteorización química moderada del área de aporte. En el diagrama A-CN-K, muchas areniscas de la Sierra Anibacachi se ubican cerca de la línea de unión de feldespatos, mientras que algunas areniscas y todas las limolitas se ubican lejos de la línea de unión de feldespatos, sugiriendo que la Zona III y Zona IV contribuyeron significativamente con los sedimentos. El que pocas areniscas se ubiquen en el límite A-K sugiere que estas areniscas fueron principalmente derivadas de la Zona II. También apoya que las areniscas hayan sufrido meteorización química baja a intensa en el área de aporte, mientras que las limolitas y lutitas han recibido materiales de rocas moderadamente meteorizadas del área de aporte. Igualmente, los valores de CIA y PIA en areniscas de la sección Rancho Búfalo sugieren que fueron derivadas de un área de aporte con meteorización baja a intensa, sin embargo, las limolitas recibieron sedimentos de la roca de origen con una meteorización química de baja a moderada. Del mismo modo, en el diagrama A-CN-K muchas areniscas y dos muestras de limolitas se ubican cerca de la línea de unión de feldespatos, mientras que algunas areniscas y una limolita se ubica lejos de dicha línea indicando que estas areniscas y limolitas fueron derivadas principalmente de la Zona III y Zona IV del perfil de meteorización en el área de origen. Lo anterior sugiere que estas areniscas y limolitas recibieron materiales de rocas afectadas por una meteorización química de un grado bajo a moderado.

Se utilizaron diagramas discriminatorios tectónico multidimensionales para interpretar los ambientes tectónicos de las rocas de origen. Las areniscas de la Sierra Anibacachi se ubicaron en los campos de arco, rift y colisión del diagrama discriminatorio tectónico multidimensional, mientras que limolitas se ubican en los campos de rift y colisión, y lutitas en el campo de colisión. Igualmente, la mayoría de areniscas y limolitas de la sección Rancho Búfalo se ubican en el campo de arco, mientras que algunas areniscas se ubican en el campo de colisión. Los resultados obtenidos de los diagramas discriminatorios tectónicos multidimensionales son consistentes con la evolución tectónica regional del área de estudio.

\section{AGRADECIMIENTOS}

Agradecemos el apoyo otorgado por la Universidad Nacional Autónoma de México a través del Proyecto PAPIIT No. IN112214-3. Este trabajo representa parte de la tesis de Maestría del autor Erik Ramírez-Montoya del posgrado en Ciencias Geología, de la Universidad de Sonora, quien desea reconocer al CONACyT por la beca otorgada para la realización de sus estudios. Agradecemos al Sr. Rufino Lozano Santa-Cruz y a la Sra. Patricia Girón García por su ayuda en el análisis de óxidos mayores. También queremos agradecer al Sr. Pablo Peñaflor por pulverizar las rocas siliciclásticas para estudios geoquímicos.

\section{REFERENCIAS}

Agrawal, S., Verma, S.P., 2007, Comment on “Tectonic classification of basalts with classification trees” by Pieter Vermeesch (2006): Geochimica et Cosmochimica Acta 71, 3388-3390. 
Anderson, T.H., Silver, L.T., 2005, The Mojave-Sonora megashear-field and analytical studies leading to the conception and evolution of the hypothesis en Anderson, T.H., Nourse, J.A., McKee, J.W., Steiner, M.B. (eds.), The Mojave-Sonora Megashear Hypothesis: Development, Assessment, and Alternatives: Geological Society America Special Paper 393, 1-50.

Armstrong-Altrin, J.S., 2009, Provenance of sands from Cazones, Acapulco, and Bahía Kino beaches, Mexico: Revista Mexicana de Ciencias Geológicas, 26(3), 764-782.

Armstrong-Altrin, J.S., Verma, S.P., 2005, Critical evaluation of six tectonic setting discrimination diagrams using geochemical data of Neogene sediments from known tectonic settings: Sedimentary Geology, 177, 115-129.

Armstrong-Altrin, J.S., Lee, Y.I., Verma, S.P., Ramasamy, S., 2004, Geochemistry of sandstones from the upper Miocene Kudankulam Formation, southern India: Implications for provenance, weathering, and tectonic setting: Journal of Sedimentary Research, 74, 285-297.

Armstrong-Altrin, J.S., Nagarajan, R., Madhavaraju, J., Rosalez-Hoz, L., Lee, Y.I., Balaram, V., Adriana Cruz-Martínez, A., Avila-Ramírez, G., 2013, Geochemistry of the Jurassic and Upper Cretaceous shales from the Molango Region, Hidalgo, eastern Mexico: Implications for sourcearea weathering, provenance, and tectonic setting: Comptes Rendus Geoscience, 345, 185-202.

Armstrong-Altrin, J.S., Nagarajan, R., Lee, Y.I., Kasper-Zubillaga, J.J., CórdobaSaldana, L.P., 2014, Geochemistry of sands along the San Nicolas and San Carlos beaches, Gulf of California Mexico: implications for provenance and tectonic setting: Turkish Journal of Earth Science, 23, 533-558.

Armstrong-Altrin, J.S., Lee, Y.I., Kasper-Zubillaga, J.J., Trejo-Ramirez, E., 2016, Mineralogy and geochemistry of sands along the Manzanillo and El Carrizal beach areas, southern Mexico: implications for palaeoweathering, provenance, and tectonic setting: Geological Journal, DOI: 10.1002/gj2792.

Arvizu, H.E., 2012, Magmatismo Permo-Triásico en el NW de Sonora, México: Inicio de la subducción y maduración de un margen continental activo: Juriquilla, Querétaro, Centro de Geociencias, Universidad Nacional Autónoma de México, Tesis de Maestría, 250 pp.

Arvizu, H.E., Iriondo, A., Aldo Izaguirre, A., Chávez-Cabello, G., Kamenov, G.D., Solís-Pichardo, G., Foster, D.A., Lozano-SantaCruz, R., 2009, Rocas graníticas pérmicas en la Sierra Pinta, NW de Sonora, México: Magmatismo de subducción asociado al inicio del margen continental activo del SW de Norteamérica: Revista Mexicana de Ciencias Geológicas, 26(3), 709-728.

Baioumy, H.M., Ismael, I.S., 2010, Factors controlling the compositional variations among the marine and non-marine black shales from Egypt: International Journal of Coal Geology, 83, 35-45.

Bhatia, M.R., 1983, Plate tectonics and geochemical composition of sandstones: Geology, 91, 611-627.

Bhatia, M.R., Crook, K.A.W., 1986, Trace element characteristics of graywackes and tectonic setting discrimination of sedimentary basins: Contribution to Mineralogy and Petrology, 92, 181-193.

Bhat, M.I., Ghosh, S.K., 2001, Geochemistry of 2.51Ga old Rampur Group pelites, western Himalayas: Implications from their provenance and weathering: Precambrian Research, 108, 1-16, https://doi.org/10.1016/ S0301-9268(00)00139-X.

Bhuiyan, M.A.H., Rahman, M.J.J., Dampare, S.B., Suzuki, S., 2011, Provenance, tectonics and source weathering of modern fluvial sediments of the Brahmaputra -Jamuna River, Bangladesh: inference from geochemistry: Journal of Geochemical Exploration, 111, 113-137.

Blanco, G., Germs, G.J.B., Rajesh, H.M., Chemale Jr., F., Dussin, I.A., Justino, D., 2011, Provenance and paleogeography of the Nama Group (Ediacaran to early Palaeozoic, Namibia): petrography, geochemistry and $\mathrm{U}-\mathrm{Pb}$ detrital zircon geochronology: Precambrian Research, 187, 15-32.

Borges, J.B., Huh, Y., Moon, S., Noh, H., 2008, Provenance and weathering control on river bed sediments of the eastern Tibetan Plateau and the Russian Far East: Chemical Geology, 254, 52-72.

Breyer, J.A., Bart, H.A., 1978, The composition of fluvial sands in a temperate semiarid region; Journal of Sedimentary Petrology, 48, 1311-1320.

Busby-Spera, C.J., 1988, Speculative tectonic model for the early Mesozoic arc of the southwest Cordilleran United States: Geology, 16, 1121-1125.

Busby-Spera, C.J., Mattinson, J.M., Riggs, N.R., Schermer, E.R., 1990, The Triassic-Jurassic magmatic arc in the Mojave-Sonoran Deserts and the
Sierran-Klamath region; Similarities and differences in paleogeographic evolution, en Harwood, D.S., Miller, M.M. (eds.), Paleozoic and early Mesozoic paleogeographic relations; Sierra Nevada, Klamath Mountains, and related terranes: Geological Society of America Special Paper 255 325-337.

Caja, M.A., Marfil, R., Lago, M., Salas, R., Ramseyer, K., 2007, Provenance discrimination of Lower Cretaceous synrift sandstones (eastern Iberian Chain, Spain): constraints from detrital modes, heavy minerals, and geochemistry, en Arribas, J., Critelli, S., Johnsson, M.J. (eds.), Sedimentary Provenance and Petrogenesis: Perspectives from Petrography and Geochemistry: Geological Society of America Special Paper, 420, 181-197.

Caracciolo, L., Von Eynatten, H., Tolosana-Delgado, R., Critelli, S., Manetti, P., Marchev, P., 2012, Petrological, geochemical, and statistical analysis of Eocene-Oligocene sandstones of the Western Thrace basin, Greece and Bulgaria: Journal of Sedimentary Research, 82, 482-498.

Chakrabarti, R., Abanda, P.A., Hannigan, R.E., Basu, A.R., 2007a, Effects of diagenesis on the Nd-isotopic composition of Black Shales from the 420 Ma Utica Shale Magnafacies: Chemical Geology, 244, 221-231.

Chakrabarti, R., Basu, A.R., Chakrabarti, A., 2007b, Trace element and Ndisotopic evidence for sediment sources in the mid-Proterozoic Vindhyan Basin, central India: Precambrian Research, 159, 260-274.

Condie, K. C., 1993, Chemical composition and evolution of the upper continental crust: contrasting results from surface samples and shales: Chemical geology, 104, 1-37.

Condie, K., DeMalas, J., 1985, The Pinal Schist: an early Proterozoic quartz wacke association in southern California: Precambrian Research, 27, 337-356.

Condie, K.C., Lee, D., Farmer, G.L., 2001, Tectonic setting and provenance of the Neoproterozoic Uinta Mountain and Big Cottonwood Groups northern Utah: contrains from geochemistry, Nd isotopes, and detrital modes: Sedimentary Geology, 141-142, 443-464.

Cox R., Lowe, D.R., 1995, A conceptural review of regional scale controls on the composition of clastic sediment and the co-evolution of continental blocks and their sedimentary cover: Journal of Sedimentary Research, 65, 1-12.

Cox, R., Lowe, D.R., Cullers, R.L., 1995, The influence of sediment recycling and basement composition on evolution of mudrock chemistry in the southwestern United States: Geochimica et Cosmochimica Acta, 59, 2919-2940.

Cullers, R.L., 1994, The controls on the major and trace element variation of shales, siltstones and sandstones of Pennsylvanian-Permian age from uplifted continental blocks in Colorado to platform sediment in Kansas, USA: Geochimica et Cosmochimica Acta, 58, 4955-4972.

Cullers, R.L., 2000, The geochemistry of shales, siltstones and sandstones of Pennsylvanian- Permian age, Colorado, U.S.A. Implications for provenance and metamorphic studies, Lithosphere, 51, 181-203.

Cullers, R.L., Podkovyrov, V.N., 2000, Geochemistry of the Mesoproterozoic Lakhanda shales in southeastern Yakutia, Russia: implications for mineralogical and provenance control, and recycling: Precambrian Research, 104, 77-93.

DaPeng, L., YueLong, C., Zhong, W., Yu, L., Jian, Z., 2012, Paleozoic sedimentary record of the Xing- Meng Orogenic Belt, Inner Mongolia: Implications for the provenances and tectonic evolution of the Central Asian Orogenic Belt: Chinese Science Bulletin, 57, 776-785.

Das, B.K., Haake B.G., 2003, Geochemistry of Rewalsar Lake sediment, Lesser Himalaya, India: implications for source-area weathering, provenance and tectonic setting: Geoscience Journal, 7, 299-312.

Dickinson, W.R., Suczek, C., 1979, Plate tectonics and Sandstone Compositions: American Association of Petroleum Geologist Bulletin, 63, 2164-2182.

Dickinson, W.R., Beard, L.S., Brakenridge, G.R., Erjavec, J.L., Ferguson, R.C., Inman, K.F., Knepp, R.A., Lindberg, F.A., Ryberg, P.T., 1983, Provenance of North American Phanerozoic sandstones in relation to tectonic setting: Geological Society of American Bulletin, 94, 222-235.

Dickinson, W.R., Klute, M.A., Swift, P.N., 1986, The Bisbee Basin and its bearing on Late Mesozoic paleogeographic and paleotectonic reconstructions between the Cordilleran and Caribbean regions, en Abbot, P.L. (ed.), Cretaceous stratigraphy of western North America: Society of Economic Paleontologists and Mineralogists, Pacific Section, Field Trip Guidebook 46, 51-62.

Fedo, C.M., Nesbitt, H.W., Young, G.M., 1995, Unravelling the effects of 
potassium metasomatism in sedimentary rocks and paleosoils, with implications for paleoweathering conditions and provenance: Geology, 23, 921-924.

Fedo, C.M., Eriksson, K., Krogstad, E.J., 1996, Geochemistry of shale from the Archaean ( 3.0 Ga) Buhwa Greenstone belt, Zimbabwe: Implications for provenance and source area weathering: Geochimica et Cosmochimica Acta, 60, 1751-1763.

Fedo, C.M., Young, G.M., Nesbitt, H.W., Hanchar, J.M., 1997, Potassic and sodic metasomatism in the southern province of the Canadian Shield: evidence from the Paleoproterozoic Serpent Formation, Huronian Supergroup, Canada: Precambrian Research, 84, 17-36.

Feng, R., Kerrich, R., 1990, Geochemistry of fine-grained clastic sediments in the Archean Abitibi greenstone belt, Canada: Implications for provenance and tectonic setting: Geochimica et Cosmochimica Acta, 54, 1061-1081.

Franzinelli, E., Potter, P.E., 1983, Petrology, chemistry and texture of modern river sands, Amazon river system: Journal of Geology, 91, 23-29.

Getaneh, W., 2002, Geochemistry provenance and depositional tectonic setting of the Adigrat Sandstone northern Ethiopia: Journal of African Earth Sciences, 35, 185-198.

Ghosh, S., Sarkar, S., Ghosh, P., 2012, Petrography and major element geochemistry of the Permo-Triassic sandstones, central India: implications for provenance in an intracratonic pull-apart basin: Journal of Asian Earth Sciences, 43, 207-240.

Gibbs, A.K., Montgomery, D.W., O’Day, P.A., Ersler, E.A., 1986, The ArcheanProterozoic transition: evidence from geochemistry of metasedimentary rocks of Guyana and Montana: Geochimica et Cosmochimica Acta, 50, 2125-2141.

Goetze, J., 1998, Geochemistry and provenance of the Altendorf feldspathic sandstone in the Middle Bunter of the Thuringian basin (Germany): Chemical Geology, 150, 43-61.

González-León, C.M., 1994, Early Cretaceous tectono-sedimentary evolution of the southwestern margin of the Bisbee Basin: Revista Mexicana de Ciencias Geológicas, 11(2), 139-146.

Girty, G.H., Barber, R.W., Knaack, C., 1993, REE, Th, and SC evidence for the depositional setting and source rock characteristics of the Quanz Hill chert, Sierra Nevada, California, en Johnsson, M.J., Basu, A. (eds.), Processes Controlling the Composition of Clastic Sediments: Geological Society of America Special Paper, 284, 109-119.

Guo, Q., Xiao,W.,Windley, B.F., Mao, Q., Han, C., Qu, J., Ao, S., Li, J., Song, D., Yong, Y., 2011, Provenance and tectonic settings of Permian turbidites from the Beishan Mountains, NW China: implications for the Late Paleozoic accretionary tectonics of the southern Altaids: Journal of Asian Earth Sciences, 49, 54-68.

Harnois, L., 1988, The CIW index: A new chemical index of weathering: Sedimentary Geology, 55, 319-322.

Herron, M.M., 1988, Geochemical classification of terrigenous sands and shales from core or log data: Journal of Sedimentary Research, 58, 820-829.

Huntsman-Mapila, P., Kampunzu, A.B., Vink, B., Ringrose, S., 2005, Cryptic indicators of provenance in the geochemistry of the Okavango Delta sediments, NW Botswana: Sedimentary Geology, 174, 123-148.

Iriondo, A., Premo, W.R., Martínez-Torres, L.M., Budahn, J.R., Atkinson, W.W. Jr., Siems, D.F., Guarás-González, B., 2004, Isotopic, geochemical and temporal characterization of Proterozoic basement rocks in the Quitovac region, northwestern Sonora, Mexico: Implications for the reconstruction of the southwestern margin of Laurentia: Geological Society of America Bulletin, 116, 154-170.

Jacques-Ayala, C., 1992, Stratigraphy of the Lower Cretaceous Cintura Formation, Sierra El Chanate, northwestern Sonora, México: Universidad Nacional Autónoma de México, Instituto de Geología, Revista, 10, 129-136.

Jacques-Ayala, C., 1995, Paleogeography and provenance of the Lower Cretaceous Bisbee Group in the Caborca-Santa Ana area, northwestern Sonora, en Jacques-Ayala, C., González-León, C.M., Roldán-Quintana, J. (eds.), Studies on the Mesozoic of Sonora and Adjacent Areas: Geological Society of America Special Paper, 301, 9-98.

Jin, Z., Li, F., Cao, J., Wang, S., Yu, J., 2006, Geochemistry of Daihai lake sediments, Inner Mongolia, north China: implications for provenance, sedimentary sorting, and catchment weathering: Geomorphology, 80, 147-163.

Johnsson, M.J., Stallard, R.F., Lundberg, N., 1991, Controls on the composition of fluvial sands from a tropical weathering environment: sands of the Orinoco river drainage basin, Venezuela and Colombia: Geological Society of America Bulletin, 103, 1622-1647.

Lahtinen, R., 2000, Archaean-Proterozoic transition: geochemistry, provenance and tectonic setting of metasedimentary rocks in central Fennoscandian Shield, Finland: Precambrian Research, 104, 147-174.

Lachance, G.R., Traill, R.J., 1966, A practical solution to the matrix problem in $\mathrm{X}$-ray analysis, I. Method: Canadian Spectroscopy, 11, 43-48.

Lawton, T.F., Molina-Garza, R.S., 2014, U-Pb geochronology of the type Nazas Formation and superjacent strata, northeastern Durango, Mexico: Implications of a Jurassic age for continental-arc magmatism in northcentral Mexico: Geological Society of America Bulletin, 126, 1181-1199.

Madhavaraju, J., 2015, Geochemistry of Late Cretaceous sedimentary rocks of the Cauvery Basin, South India: Constraints on paleoweathering, provenance and end Cretaceous environments, en Ramkumar, M. (ed.), Chemostratigraphy, Concepts, Techniques and Applications: Elsevier, 185-214.

Madhavaraju, J., Lee, Y.I., 2010, Influence of Deccan volcanism in the sedimentary rocks of Late Maastrichtian-Danian age of Cauvery basin Southeastern India: constraints from geochemistry: Current Science, 98, 528-537.

Madhavaraju, J., Ramasamy, S., 2002, Petrography and geochemistry of Late Maastrichtian - Early Paleocene sediments of Tiruchirapalli Cretaceous, Tamil Nadu- Paleoweathering and provenance implications: Journal of the Geological Society of India, 59, 133-142.

Madhavaraju J., Milu T., Lee, Y.I., Balaram V., Ramasamy S., Carranza-Edwards A., Ramachandran A., 2016a, Provenance and tectonic settings of sands from Puerto Peñasco, Desemboque and Bahia Kino beaches, Gulf of California, Sonora, Mexico: Journal of South American Earth Sciences, 71, 262-275.

Madhavaraju, J., Ramírez-Montoya, E., Monreal, R., González-León, C.M., Pi-Puig, T., Espinoza-Maldonado, I.G., Grijalva-Noriega, F.J, 2016b, Paleoclimate, paleoweathering and paleoredox conditions of Lower Cretaceous shales from the Mural Limestone, Tuape section, northern Sonora, Mexico: Constraints from clay mineralogy and geochemistry: Revista Mexicana de Ciencias Geológicas, 33(1), 34-48.

Maynard, J.B., Valloni, R., Yu, H., 1982, Composition of modern deep sea sands from arc-related basins: Geological Society of London Special Publication, $10,551-561$

McLennan, S.M., 1993, Weathering and global denudation: The Journal of Geology, 101, 295-303.

McLennan, S.M., Taylor, S.R., 1991, Sedimentary rocks and crustal evolution: tectonic setting and secular trends: The Journal of Geology, 1-21.

McLennan, S.M., Taylor, S.R., McCulloch, M.T., Maynard, J.B., 1990, Geochemical and $\mathrm{Nd} / \mathrm{Sr}$ isotopic composition of deep-sea turbidites: crustal evolution and plate tectonic associations: Geochimica et Cosmochimica Acta, 54, 2015-2050.

McLennan, S.M., Hemming, S., McDaniel, D.K., Hanson, G.N., 1993, Geochemical approaches to sedimentation, provenance and tectonics: Geological Society of America, Special Paper, 284, 21-40.

McLennan, S.M., Hemming, S.R., Taylor, S.R., Eriksson, K.A., 1995, Early Proterozoic crustal evolution: geochemical and $\mathrm{Nd}-\mathrm{Pb}$ isotopic evidence from metasedimentary rocks, southwestern North America: Geochimica et Cosmochimica Acta, 59, 1153-1177.

Mondal, M.E.A., Wani, H., Mondal, B., 2012, Geochemicalsignature of provenance, tectonics and chemical weathering in the Quaternary flood plain sediments of the Hindon River, Gangetic plain, India: Tectonophysics, 566, 87-94.

Monreal, R., Valenzuela, M., González-León, C., 1994, A revision of the stratigraphic nomenclature for the Cretaceous of northern Sonora, and some paleogeographic implications: Boletín del Departamento de Geología, Numero especial del XX Aniversario de la Universidad de Sonora, 11, 171-190.

Murray, R.W., 1994, Chemical criteria to identify the depositional environment of chert: general principles and applications: Sedimentary Geology, 90, 213-232.

Nagarajan, R., Armstrong-Altrin, J.S., Nagendra, R., Madhavaraju, J., Moutte, J., 2007, Petrography and geochemistry of terrigenous sedimentary rocks in the Neoproterozoic Rabanpalli Formation, Bhima Basin, southern India: 
Implications for paleoweathering condition, provenance, and source rock composition: Journal of the Geological Society of India, 70, 297-312.

Nesbitt, H.W., Young, G.M., 1982, Early Proterozoic climates and plate motions inferred from major element chemistry of lutitas: Nature, 299, 715-717.

Nesbitt, H.W., Young, G.M., 1984, Prediction of some weathering trends of plutonic and volcanic rocks based on thermodynamic and kinetic considerations: Geochimica et Cosmochimica Acta, 48, 1523-1534.

Nesbitt, H.W., Young, G.M., 1989, Formation and diagenesis of weathering profiles: The Journal of Geology, 129-147.

Nesbitt, H.W., Markovics, G., Price, R.C., 1980, Chemical processes affecting alkalies and alkaline earths during continental weathering: Geochimica et Cosmochimica Acta, 44, 1659-1666.

Nesbitt, H.W., Young, G.M., McLennan, S.M., Keays, R.R., 1996, Effects of chemical weathering and sorting on the petrogenesis of siliciclástic sediments, with implications for provenance studies: The Journal of Geology, 104, 525-542.

Nesbitt, H.W., Fedo, C.M., Young, G.M., 1997, Quartz and feldspar stability, steady and non-steady-state weathering, and petrogenesis of siliciclastic sands and muds: The Journal of Geology, 105, 173-191.

Ohta, T., 2004, Geochemistry of Jurassic to earliest Cretaceous deposits in the Nagato Basin, SW Japan: implication of factor analysis to sorting effects and provenance signatures: Sedimentary Geology, 171, 159-180.

Page, W.R., Gray, F., Iriondo, A., Miggins, D., Blodgett, R.B., Maldonado, F., Miller, R.J., 2010, Stratigraphy and Mesozoic-Cenozoic tectonic history of northern Sierra Los Ajos and adjacent areas, Sonora, Mexico: Journal of South American Earth Sciences, 29, 557-571.

Pe-Piper, G., Triantafyllidis, S., Piper, D.J.W., 2008, Geochemical identification of clastic sediment provenance from known sources of similar geology: the Cretaceous Scotian basin, Canada: Journal of Sedimentary Research, 78, 595-607.

Peryam, T.C., Lawton, T.F., Amato J.M., González-León, C.M., David J. Mauel, D.J., 2012, Lower Cretaceous strata of the Sonora Bisbee Basin: A record of the tectonomagmatic evolution of northwestern Mexico: Geological Society of America Bulletin, 124, 532-548.

Pollack, J.M., 1961, Significance of compositional and textural properties of South Canadian River channel sands, New Mexico, Texas, and Oklahoma: Journal of Sedimentary Petrology, 31, 15-37.

Ramachandran, A., Madhavaraju, J., Ramasamy, S., Lee, Y.I., Rao, S., Chawngthu, D.L., Velmurugan, K., 2016, Geochemistry of the Proterozoic clastic rocks of Kerur Formation of Kaladgi-Badami Basin, Northern Karnataka, South India: Implications for paleoweathering and provenance: Turkish Journal of Earth Sciences, 25, 126-144.

Ransome, F.L., 1904, The geology and ore deposits of the Bisbee quadrangle Arizona: U.S. Geological Survey, Professional Paper, 21, 168 pp.

Rodríguez-Castañeda, J.L., Anderson, T.H., 2011, El arco magmático jurásico en Sonora, México-distribución, edades y ambiente tectónico, en Calmus, T. (ed.), Panorama de la Geología de Sonora, México: Universidad Nacional Autónoma de México, Instituto de Geología, Boletín, 118, 81-111.

Roser, B., Korsch, R., 1985, Plate tectonics and geochemical composition of sandstones: a discussion: Journal of Geology, 93, 81-84.

Roser, B., Korsch, R., 1986, Determination of tectonic setting of sandstonemudstone suites using $\mathrm{SiO}_{2}$ content and $\mathrm{K}_{2} \mathrm{O} / \mathrm{Na}_{2} \mathrm{O}$ ratio: The Journal of Geology, 94, 635-650.

Roser, B., Korsch, R., 1988, Provenance signatures of sandstone-mudstone suites determined using discrimination function analysis of major-element data: Chemical Geology, 67, 119-139.

Rudnick, R.L., Gao, G., 2004, Composition of the continental crust, en Rudnick, R.L. (ed.), The Crust: Treatise on Geochemistry: Amsterdam, The Netherlands, Elsevier, 3, 1-64.

Ryan, K.M., Williams, D.M., 2007, Testing the reliability of discrimination diagrams for determining the tectonic depositional environment of ancient sedimentary basins: Chemical Geology, 242, 103-125.

Solari, L.A., González-León, C.M., Ortega-Obregón, C., Valencia-Moreno, M., Rascón-Heimpel, M.A., 2017, The Proterozoic of NW Mexico revisited: $\mathrm{U}-\mathrm{Pb}$ geochronology and $\mathrm{Hf}$ isotopes of Sonoran rocks and their tectonic implications: International Journal of Earth Sciences, DOI 10.1007/ s00531-017-1517-2.

Spalletti, L.A., Merodio, J.C., Matheos, S.D., 1991, Geoquímica y significado tectónico-depositacional de pelitas y margas Cretácico-Terciarias.
Noroeste de la Patagonia, República Argentina: Revista Geológica de Chile, 20, 3-13.

Spalletti, L.A., Merodio, J.C., Matheos, S.D., 1992, Composición, procedencia y marco tectónico de sedimentitas eojurásicas (Formación Nestares) del extremo sudeste de la Cuenca Neoquina: Asociación Geológica Argentina Revista, 43, 227-286.

Spalletti, L.A., Limarino, C.O., Pinol, F.C., 2012, Petrology and geochemistry of Carboniferous siliciclastics from the Argentine Frontal Cordillera: A test of methods for interpreting provenance and tectonic Setting: Journal of South America Earth Science, 36, 32-54.

Sreenivas, B., Srinivasan, R., 1994, Identification of paleosols in the Precambrian metapelitic assemblages of peninsular India-A major element geochemical approach: Current Science, 67, 89-94.

Sun, L., Gui, H., Chen, S., 2012, Geochemistry of sandstones from the Neoproterozoic Shijia Formation, northern Anhui Province, China: implications for provenance, weathering and tectonic setting: Chemie der Erde, 77, 253-260.

Taliaferro, N.L., 1933, An occurrence of Upper Cretaceous sediment in northern Sonora, Mexico: Journal of Geology, 41, 12-37.

Tapia-Fernandez, H.J., Armstrong-Altrin, J.S., Selvaraj, K., 2017, Geochemistry and $\mathrm{U}-\mathrm{Pb}$ geochronology of detrital zircons in the Brujas beach sands, Campeche, Southwestern Gulf of Mexico, Mexico: Journal of South American Earth Sciences, 76, 346-361.

Taylor, S.R., McLennan, S.M., 1985, The continental crust: its composition and evolution: Blackwell, Oxford, 312 pp.

Taylor, S.R., McLennan S.M., 1995, The geochemical evolution of the continental crust: Reviews of Geophysics, 33, 241-265.

Thomas, C.W., Aitchison, J., 2005, Compositional data analysis of geological variability and process: a case study: Mathematical Geology, 37, 753-772.

Tobia, F.H., Shangola, S.S., 2016, Mineralogy, geochemistry, and depositional environment of the Beduh Shale (Lower Triassic), Northern Thrust Zone, Iraq: Turkish Journal of Earth Sciences, 25, 367-391.

Verma, S.P., 2010, Statistical evaluation of bivariate, ternary and discriminant function tectonomagmatic discrimination diagrams: Turkish Journal of Earth Science, 19, 185-238.

Verma, S.P., 2012, Application of multi-dimensional discrimination diagrams and probability calculations to acid rocks from Portugal and Spain: Comunicações Geológicas, 99, 79-93.

Verma, S.P., Armstrong-Altrin, J.S., 2013, New multi-dimensional diagrams for tectonic discrimination of siliciclastic sediments and their application to Precambrian basins: Chemical Geology, 355, 117-133.

Verma, S.P., Armstrong-Altrin, J.S., 2016, Geochemical discrimination of siliciclastic sediments from active and passive margin settings: Sedimentary Geology, 332, 1-12.

von Eynatten, H., Dunkl, I., 2012, Assessing the sediment factory: the role of single grain analysis: Earth Science Review, 115, 97-120.

Weltje, G.J., 2006, Ternary sandstone composition and provenance: An evaluation of the 'Dickinson model': Geological Society London Special Publication, 264, 79-99.

Weltje, G.J., 2012, Quantitative models of sediment generation and provenance: state of the art and future developments: Sedimentary Geology, 280, 4-20.

Wronkiewicz, D.J., Condie, K.C., 1987, Geochemistry of Archaean shales form the witwatersrand Supergroup, South Africa. Source-area weathering and provenance: Geochimica et Cosmochimica Acta, 51, 2401-2416.

Wronkiewicz, D.J., Condie, K.C., 1989, Geochemistry and provenance of sediments from the Pongola Supergroup, South Africa: evidence for a 3.0 Ga old continental craton: Geochimica et Cosmochimica Acta, 53, 537-549.

Wronkiewicz, D., Condie, K., 1990, Geochemistry and mineralogy of sediments from the Ventersdorp and Transvaal Supergroups, South Africa. Cratonic evolution during the early Proterozoic: Geochimica et Cosmochimica Acta, 54, 343-354.

Zaid, S.M., 2012, Provenance, diagenesis, tectonic setting and geochemistry of Rudies sandstone (Lower Miocene), Warda Field, Gulf of Suez, Egypt: Journal of African Earth Sciences, 66-67, 56-71.

Manuscrito recibido: marzo 15, 2017

Manuscrito corregido recibido: noviembre 22, 2017

Manuscrito aceptado: noviembre 29, 2017 